\title{
The Prolyl-tRNA Synthetase Inhibitor Halofuginone Inhibits SARS-CoV-2 Infection
}

Daniel R. Sandoval ${ }^{1,2, \#}$, Thomas Mandel Clausen ${ }^{2,3, \#}$, Chelsea Nora ${ }^{1, \#}$, Adam P. Cribbs ${ }^{4}$, Andrea Denardo $^{1,5}$, Alex E. Clark ${ }^{1,2}$, Aaron F. Garretson ${ }^{1}$, Joanna K.C. Coker ${ }^{1,6}$, Anoop Narayanan ${ }^{6}$, Sydney A. Majowicz ${ }^{6}$, Martin Philpott ${ }^{4}$, Catrine Johansson ${ }^{4}$, James E. Dunford ${ }^{4}$, Charlotte B. Spliid ${ }^{2}$, Gregory J. Golden ${ }^{2,8}$, N. Connor Payne ${ }^{9,10}$, Mark A. Tye ${ }^{9,11}$, Cameron J. Nowell' ${ }^{12}$ Eric R. Griffis $^{13}$, Ann Piermatteo ${ }^{1}$, Kaare V. Grunddal ${ }^{1}$, Thibault Alle ${ }^{14}$, Jason A. Magida ${ }^{15}$, Blake M. Hauser $^{16}$, Jared Feldman ${ }^{17}$, Timothy M. Caradonna ${ }^{18}$, Yuan $\mathrm{Pu}^{19}$, Xin Yin ${ }^{17}$, Rachael N. McVicar $^{6,18,19}$, Elizabeth M. Kwong ${ }^{6,18,19}$, Ryan J. Weiss ${ }^{2}$, Michael Downes $^{15}$, Sotirios Tsimikas ${ }^{1}$, Aaron G. Smidt ${ }^{16,20}$, Carlo Ballatore ${ }^{14}$, Karsten Zengler ${ }^{6,21,22}$, Ron M. Evans ${ }^{15,23}$, Sumit K. Chanda $^{17}$, Ben A. Croker ${ }^{6}$, Sandra L. Leibel ${ }^{6,18,19}$, Joyce Jose ${ }^{7}$, Ralph Mazitschek ${ }^{9,24,25}$, Udo Oppermann ${ }^{4,26}$, Jeffrey D. Esko ${ }^{2,27}$, Aaron F. Carlin ${ }^{1,{ }^{*}}$, Philip L.S.M. Gordts ${ }^{1,27,{ }^{*}}$

${ }^{1}$ Department of Medicine, University of California, San Diego, La Jolla, CA 92093, USA. ${ }^{2}$ Department of Cellular and Molecular Medicine, University of California, San Diego, La Jolla, CA 92093, USA. ${ }^{3}$ Department for Immunology and Microbiology, Faculty of Health and Medical Sciences, University of Copenhagen and Department of Infectious Disease, Copenhagen, University Hospital, 2200 Copenhagen, Denmark. ${ }^{4}$ Botnar Research Centre, NIHR Biomedical Research Centre, University of Oxford, OX3 7LD, UK. ${ }^{5}$ Department of Molecular and Translational Medicine, University of Brescia, Brescia, Italy. ${ }^{6}$ Department of Pediatrics, University of California, San Diego, La Jolla, CA 92037, USA. ${ }^{7}$ Department of Biochemistry and Molecular Biology, The Huck Institutes of the Life Sciences, The Pennsylvania State University, University Park, PA, 16802, USA. ${ }^{8}$ Biomedical Sciences Graduate Program, University of California San Diego, La Jolla, CA, USA. ${ }^{9}$ Center for Systems Biology, Massachusetts General Hospital, Boston, MA, 02114, USA. ${ }^{10}$ Department of Chemistry \& Chemical Biology, Harvard University, Cambridge, 
MA, 02114, USA. ${ }^{11}$ Harvard Graduate School of Arts and Sciences, Cambridge, MA, 02138, USA.

${ }^{12}$ Monash Institute of Pharmaceutical Sciences, Victoria 3052, Australia. ${ }^{13}$ Nikon Imaging Center, University of California, San Diego, La Jolla, CA 92037, USA. ${ }^{14}$ Skaggs School of Pharmacy and Pharmaceutical Sciences, University of California, San Diego, La Jolla, California, USA. ${ }^{15}$ Gene Expression Laboratory, Salk Institute for Biological Studies, La Jolla, CA, USA. ${ }^{16}$ Ragon Institute of MGH, MIT and Harvard, Cambridge, MA 02139, USA. ${ }^{17}$ Immunity and Pathogenesis Program, Infectious and Inflammatory Disease Center, Sanford Burnham Prebys Medical Discovery Institute, La Jolla, CA 92037, USA. ${ }^{18}$ Sanford Burnham Prebys Medical Discovery Institute, La Jolla, CA 92037, USA. ${ }^{19}$ Sanford Consortium for Regenerative Medicine, La Jolla, CA 92037, USA. ${ }^{20}$ Department of Microbiology, Harvard Medical School, Cambridge, MA, 02115 USA. ${ }^{21}$ Department of Bioengineering, University of California, San Diego, 9500 Gilman Drive, La Jolla, CA 92093-0412, USA. ${ }^{22}$ Center for Microbiome Innovation, University of California, San Diego, 9500 Gilman Drive, La Jolla, CA, 92093-0436, USA. ${ }^{23}$ Howard Hughes Medical Institute, Salk Institute for Biological Studies, La Jolla, CA, USA. ${ }^{24}$ Harvard T.H. Chan School of Public Health, Cambridge, MA, 02115, USA. ${ }^{25}$ Broad Institute of MIT and Harvard, Cambridge, MA, 02142 , USA. ${ }^{26}$ Centre for Medicines Discovery, University of Oxford, UK. ${ }^{27}$ Glycobiology Research and Training Center, University of California, San Diego, La Jolla, CA 92093, USA.

${ }^{\#}$ Contributed equally to the work.

*To whom correspondence should be addressed: Philip L.S.M. Gordts, Department of Medicine, University of California, La Jolla, CA 92093, USA; +1(858)246-0994, pgordts@health.ucsd.edu, Aaron F. Carlin, Department of Medicine, University of California, La Jolla, CA 92093, USA; +1(858)246-3261, acarlin@health.ucsd.edu 


\section{[Summary Paragraph]}

We identify the prolyl-tRNA synthetase (PRS) inhibitor halofuginone ${ }^{1}$, a compound in clinical trials for anti-fibrotic and anti-inflammatory applications ${ }^{2}$, as a potent inhibitor of SARS-CoV-2 infection and replication. The interaction of SARS-CoV-2 spike protein with cell surface heparan sulfate (HS) promotes viral entry ${ }^{3}$. We find that halofuginone reduces HS biosynthesis, thereby reducing spike protein binding, SARS-CoV-2 pseudotyped virus, and authentic SARS-CoV-2 infection. Halofuginone also potently suppresses SARS-CoV-2 replication post-entry and is 1,000-fold more potent than Remdesivir ${ }^{4}$. Inhibition of HS biosynthesis and SARS-CoV-2 infection depends on specific inhibition of PRS, possibly due to translational suppression of proline-rich proteins. We find that pp1a and pp1ab polyproteins of SARS-CoV-2, as well as several HS proteoglycans, are proline-rich, which may make them particularly vulnerable to halofuginone's translational suppression. Halofuginone is orally bioavailable, has been evaluated in a phase I clinical trial in humans and distributes to SARS-CoV-2 target organs, including the lung, making it a near-term clinical trial candidate for the treatment of COVID-19. 


\section{[MAIN TEXT]}

Severe acute respiratory syndrome-related coronavirus 2 (SARS-CoV-2), the causative agent of coronavirus disease 2019 (COVID-19), has swept across the globe causing 32.1 million infections and 980,339 confirmed deaths as of September $24^{\text {th }} 2020^{5}$. In May 2020, the US Food and Drug Administration (FDA) granted an Emergency Use Authorization (EUA) for Remdesivir (RDV, GS5734) for the treatment of hospitalized patients with severe COVID-196. In the preliminary report of the ACTT-1 clinical trial subgroup analysis, Remdesivir significantly reduced time to recovery only in patients who were on low-flow oxygen at baseline ${ }^{4}$. This finding suggested that Remdesivir treatment in COVID-19 may provide greater benefit if started before the development of severe disease $^{7,8}$. However, data from multiple trials suggests that Remdesivir provides modest clinical benefit compared with standard of care ${ }^{4,9,10}$. Furthermore, Remdesivir must be administered intravenously, which functionally prevents its use in pre-symptomatic or early symptomatic mild disease. Convalescent plasma from individuals who have recovered from COVID-19 has also been granted EUA for hospitalized patients with COVID-19 but efficacy data from randomized trials are needed. Hence, there is an urgent unmet clinical need for antiviral therapeutics that can reduce COVID-19 associated morbidity and mortality and that optimally could be administered early after symptom onset to prevent the development of severe respiratory disease $\mathrm{e}^{11,12}$.

SARS-CoV-2 entry into lung upper airway epithelial cells depends on ACE2, TMPRSS2, and cell surface heparan sulfate $(\mathrm{HS})^{3,13,14}$. Blocking the interaction of cell surface HS and spike protein attenuate SARS-CoV-2 binding and SARS-CoV-2 infection ${ }^{3}$. In this report we identified halofuginone, a synthetic analog of the natural product febrifugine that is derived from Dichroa febrifuga (one of the 50 fundamental herbs of traditional Chinese medicine), as a potent inhibitor of HS biosynthesis and SARS-CoV-2 infection ${ }^{1,15}$. We demonstrate that halofuginone reduces HS biosynthesis, thereby decreasing SARS-CoV-2 spike protein binding to cells, infection with SARSCoV-2 spike protein pseudotyped VSV virus, and infection of authentic SARS-CoV-2. Additionally, 
halofuginone inhibited authentic SARS-CoV-2 replication post-entry. Inhibition of prolyl-tRNA synthetase (PRS) activity was responsible for both the HS-dependent and the HS-independent antiviral properties of halofuginone. These findings identify halofuginone as a translational regulator of cell surface HS biosynthesis and a potentially potent, orally bioavailable, therapeutic for the treatment and prevention of COVID-19.

\section{RESULTS}

Halofuginone Reduces SARS-CoV-2 Spike Protein Binding and Infection by SARS-CoV-2 Spike Protein Pseudotyped Virus.

To identify potential agents that attenuate SARS-CoV-2 spike protein binding, we screened a library of small-molecule antagonists of host epigenetic regulators and protein translation elements of the prolyl-tRNA synthetase complex, along with their chemically matched inactive analogs ${ }^{16-18}$. The compound library has been successfully used to investigate targets mediating anti-inflammatory or anti-proliferative effects in a variety of biological contexts ${ }^{16-18}$ and importantly, contains several FDA-approved drugs, which may facilitate rapid deployment for treatment of COVID-19 patients. Hep3B human hepatoma cells were treated with the compound library at the indicated concentrations (Fig. 1a \& Extended data Fig. 1) for $18 \mathrm{~h}$, and binding of SARS-CoV-2 spike protein was assessed using recombinant receptor binding domain (RBD; from isolate Wuhan 20) in flow cytometry (Fig. 1a). As a positive control cells were treated with a mixture of heparin lyases, which digest HS chains ${ }^{3}$. Class 1 histone deacetylase inhibitors, such as Romidepsin $(1 \mu \mathrm{M})$ or Belinostat $(5 \mu \mathrm{M})$, decreased SARS-CoV-2 RBD binding by $\sim 50 \%$ compared to excipient control (DMSO; Fig 1a). Halofuginone $(1 \mu \mathrm{M})$ reduced binding by $84 \%$, a level of reduction similar to the impact of heparin lyase treatment (Fig 1a). Halofuginone targets the prolyl-tRNA synthetase (PRS) active site of the human glutamyl-prolyl tRNA synthetase (EPRS) and has been used in clinical and preclinical studies to treat fibrotic disease and to 
attenuate hyperinflammation ${ }^{1,19}$. We then treated Vero E6 African green monkey kidney epithelial cells, Caco-2 human epithelial colorectal adenocarcinoma cells, and Calu-3 human epithelial lung adenocarcinoma cells with halofuginone. Halofuginone reduced RBD protein binding to Hep3B, Calu-3 and Caco-2 but showed very modest effects in Vero E6 cells (Fig. 1b-e). The degree of inhibition was greatest in the Calu-3 line (5-fold reduction in RBD binding; Fig. 1e). To test the effect of halofuginone on the binding of spike protein in a more native presentation, we examined the ability of halofuginone to inhibit infection of SARS-CoV-2 spike protein pseudotyped VSV in Hep3B cells. Halofuginone inhibited infection by up to $\sim 30$-fold in a dose-dependent manner (Fig. 1f). Consistent with the inability of halofuginone to reduce RBD binding to Vero E6 cells (Fig. 1c), no inhibition of infection was seen in these cells (Fig. 1g). We next tested if halofuginone inhibits the infection of authentic SARS-CoV-2 in Hep3B. Cells were treated with halofuginone at different doses for $24 \mathrm{~h}$ prior to and during infection (MOI of 0.1$)(\mathrm{Fig} .1 \mathrm{~h})^{20}$. The culture medium was collected, and the presence of virus was measured by plaque assays in Vero E6 cells. No infectious SARS-CoV-2 could be detected in the supernatants of Hep3B cells treated with halofuginone at doses greater than $50 \mathrm{nM}$ (Fig. 1h). To explore the effect of halofuginone on SARS-CoV-2 infection in a more clinically relevant model, we grew primary human bronchial epithelial cells grown at an air-liquid interface and infected them with authentic SARS-CoV-2 virus with and without halofuginone treatment. Halofuginone significantly reduced the number of SARSCoV-2 infected cells at both $10 \mathrm{nM}$ and $100 \mathrm{nM}$ without affecting cell viability (Fig. $1 \mathrm{i}-\mathrm{j}$ \& Extended data Fig. 2).

\section{Halofuginone Inhibits Heparan Sulfate Biosynthesis}

The binding of SARS-CoV-2 spike protein to cells is HS-dependent ${ }^{3}$. HS is a linear polysaccharide attached to serine residues in $\mathrm{HS}$ proteoglycans $(\mathrm{HSPGs})^{21}$. The HS polysaccharides consist of alternating residues of $\mathrm{N}$-acetylated or $\mathrm{N}$-sulfated glucosamine (GlcNAc or GlcNS) and either glucuronic acid (GlcA) or iduronic acid (IdoA) (Fig. 2a). Hence, we 
tested if halofuginone reduces spike protein binding by reducing HS presentation at the cell surface. Hep3B and Vero E6 cells were treated for $18 \mathrm{~h}$ with halofuginone and the effects on cellular HS were evaluated using the monoclonal antibody (mAb) (10E4) that recognizes a common epitope in HS (Fig. 2b \& Extended data Fig. 3). Halofuginone dose-dependently reduced 10E4 binding in Hep3B (Fig. 2c), whereas 10E4 binding increased in Vero E6 upon halofuginone treatment (Fig. 2d). This directly correlates with the level of spike RBD binding and $S$ protein pseudotyped virus infection in these cells (Fig. 1b-c). This finding suggests that halofuginone inhibits spike protein binding and SARS-CoV-2 viral attachment by altering cell surface HS content. Analysis of Hep3B cells showed that $0.5 \mu \mathrm{M}$ halofuginone reduced cell surface HS in Hep3B cells by $\sim 4$-fold (Fig. 2e). No difference was seen in the disaccharide composition following halofuginone treatment, suggesting that halofuginone affects total HS synthesis but does not alter HS specific sulfation (Fig. 2f). To test if the observed decrease in total HS was due to a decrease in availability of HSPG core proteins to carry HS, we lysed treated Hep3B cells, ran the lysates on SDS-PAGE and stained using an mAb (3G10) that recognizes a neo-epitope remaining on the proteoglycans (Fig. $2 b, g)^{22}$. The analysis revealed that halofuginone reduced the expression of core HSPGs in a dose-dependent manner (Fig. $2 g$ \& Extended data Fig. 4). These data suggest that halofuginone inhibits HS-mediated binding of the SARS-CoV-2 spike protein to cells by inhibiting the expression of HSPGs.

To broadly examine the effects of halofuginone treatment, Hep3B cells were treated with vehicle or halofuginone at 200 or $500 \mathrm{nM}$ for $6 \mathrm{~h}$ and $18 \mathrm{~h}$ and processed for RNA-sequencing (RNA-Seq). Halofuginone profoundly impacted the transcriptome ( 2700 differentially expressed genes), which increased with longer incubation time (Extended data Fig. 5a-b). Principal component analysis clearly segregated the response with respect to the duration of treatment (Extended data Fig. 5a). Analysis of halofuginone upregulated genes did not identify common pathways associated with antiviral or inflammatory responses (Extended data Fig. 5c-d $)^{23}$. Additionally, no significant difference in expression of host factors exploited by SARS-CoV-2, such 
as TMPRSS2 and ACE2, were observed (Extended data Fig. 5f-g). Gene annotation analysis of downregulated genes at $6 \mathrm{~h}$ showed that $200 \mathrm{nM}$ halofuginone altered the expression of genes involved in glycoprotein biosynthesis and proteoglycan metabolic processes (Extended data Fig. 5e) $)^{23}$. A select group of core HSPGs were downregulated at the mRNA level (Extended data Fig. 5f-g). In particular, HSPGs GPC2 and SDC1 were significantly downregulated in conjunction with the HS biosynthetic enzymes B3GAT3 and EXTL3 (Extended data Fig. 5f-g \& 6). Taken together, the data demonstrates that halofuginone suppresses the expression of proteins involved in HS and HSPG production.

\section{Halofuginone Inhibition of Spike Protein Binding to Heparan Sulfate is Independent of the Canonical Integrated Stress Response.}

Aminoacyl-tRNA-synthetases (AARS) catalyze the ATP-dependent synthesis of aminoacylated tRNAs via a two-step reaction involving an aminoacyl-adenylate intermediate with subsequent transfer to the cognate tRNAs ${ }^{24}$. Halofuginone and chemically unrelated compounds like prolyl-sulfamoyl adenosine (ProSA), specifically and potently inhibit human prolyl-tRNA synthetase (PRS) by blocking distinct portions of their ligand binding pockets (Fig. 3a-c) $)^{15,25}$. PRS inhibition can specifically suppress translation of proteins, such as collagens, that are enriched in prolines while having minimal effects on general protein synthesis ${ }^{15}$. However, PRS inhibition can also lead to GCN2-mediated activation of the Integrated Stress Response (ISR). GCN2 (gene symbol EIF2AK4) senses uncharged tRNAs and phosphorylates the eukaryotic transcription initiation factor $2 \alpha$ (elF2 $\alpha$ ), leading to a general reduction in 5'cap-mediated RNA translation and selective translation of the eukaryotic transcription factor ATF4 and its target genes (Fig. 3a) ${ }^{26}$. These genes contain structural features in their 5'UTR allowing for selective translation in the presence of Ser51 phosphorylated elF2 $\alpha$. The complexity and transient nature of the transcriptional and translational changes mediated by different elF2 $\alpha$ kinases of the ISR allows a 
cell to adapt and resolve various stress situations including amino acid starvation or unfolded protein stress ${ }^{26}$.

Interestingly, halofuginone induced a general ATF4-mediated ISR but did not activate the unfolded protein-induced ER stress response, as reported previously (Extended data Fig. 5d) ${ }^{27}$. To date, alterations in HS biosynthesis have not been identified as a hallmark of the ISR. To better understand the relationship between PRS inhibition and HS expression we tested related PRS inhibitor analogs to deconstruct the PRS pathway in relation to HS biosynthesis and spike RBD binding. Treatment with the non-cleavable and highly selective prolyl-AMP substrate analog ProSA at $5 \mu \mathrm{M}$ prevented HS presentation and spike RBD binding to similar levels as treatment with halofuginone $(500 \mathrm{nM})$, as illustrated by mAb 10E4 stain (Fig. 3c-d ${ }^{28}$. Additionally, halofuginol (HFol), a halofuginone derivative that inhibits PRS, significantly decreased $10 \mathrm{E} 4$ and spike RBD binding while the MAZ1310 negative control compound, had no effect (Fig. 3d-e) $)^{15,25}$. Collectively, the data demonstrates that PRS inhibition is sufficient to reduce HS biosynthesis and spike RBD binding (Fig. 3d-e). Halofuginone competes with proline for the PRS active site ${ }^{15}$. Addition of excess proline to the media $(4 \mathrm{mM})$ of Hep3B cells prevented the ability of halofuginone to inhibit RBD protein binding to the cells (Fig. 3f).

To determine if halofuginone suppresses HS biosynthesis by activating the ISR we coincubated halofuginone in the presence of a general inhibitor of the ISR, ISRIB (Integrated Stress Response inhibitor), selective elF2 $\alpha$ kinase inhibitors GCN2-IN-1 (GCN2i) targeting GCN2 (general control nonderepressible 2), or GSK2606414 (PERKi) targeting elF2 $\alpha$ kinase 3 (elF2AK3), also known as protein kinase R-like endoplasmic reticulum kinase (PERK) (Fig. $3 A)^{26,29}$. Neither PERKi nor ISRIB affected 10E4 or RBD binding, and neither had an effect on halofuginone inhibition (Fig. 3g-h). In contrast, GCN2i reduced 10E4 and RBD binding almost to the same extent as halofuginone activity (Fig. 3g-h), suggesting a role for GCN2 in the regulation of HS biosynthesis independent of the ISR. Neither GCN2-IN-1, GSK2606414, nor ISRIB 
reversed the halofuginone induced reduction in $10 \mathrm{E} 4$ or spike protein binding, suggesting that halofuginone does not suppress HS biosynthesis and spike binding by activating the ISR (Fig. 3gh).

PRS inhibitors can selectively modulate the translational efficiency of proline-rich proteins, such as collagens (Extended data Fig. 7). Interestingly, HSPGs, such as agrin, perlecan, collagen 18 and syndecans 1 and 3, are relatively proline-rich compared to other proteins, such as TMPRSS2, ACE2 and lysosomal or cholesterol biosynthetic proteins (Fig. 3i and Extended data Fig. 7-9; Extended data Table 1). These observations suggest that inhibition of prolyl-tRNA charging could impair production of key membrane and extracellular matrix HSPGs (Fig. 3i and 2g). Together, these data suggest that PRS inhibitors, such as halofuginone, inhibit HS and proteoglycan expression both at the translational and transcriptional level.

\section{Halofuginone Inhibits infection and Replication by Authentic SARS-CoV-2}

To determine if halofuginone inhibition of SARS-CoV-2 virus production was due to reduced viral entry or subsequent intra-host replication, Huh 7.5 cells were treated with $100 \mathrm{nM}$ halofuginone or vehicle, either before, after, or before and after infection with SARS-CoV-2. Similarly, as in Hep3B halofuginone prevented productive infection of Huh 7.5 without affecting cell viability (Fig. 4a-b, and Extended data Fig. 10). Pretreatment with halofuginone alone significantly reduced SARS-CoV-2 infection by $\sim 30$-fold (Fig. 4a). However, halofuginone added after viral infection reduced the amount of secreted infectious virions by nearly 1000-fold (Fig. 4a). Intracellular viral RNA did not change when the cells were only treated before infection as expected, but viral RNA levels dramatically decreased 10- to 100-fold when halofuginone was present after infection (Fig. 4b). These observations suggest that halofuginone inhibits SARSCoV-2 viral replication in addition to suppressing HS-dependent infection.

Halofuginone did not decrease cellular HS or the binding of recombinant RBD protein in Vero E6 cells. However, given the effects of halofuginone treatment on viral replication we 
examined the effect of halofuginone on infection by authentic SARS-CoV-2 in Vero E6 cells as well. Halofuginone completely inhibited SARS-CoV-2 infectivity at $50 \mathrm{nM}$ with an $\mathrm{IC}_{50}$ of $13 \mathrm{nM}$ as measured by immunofluorescent (IF) detection of the nucleocapsid protein and plaque assays (Fig. 4c-d \& Extended data Fig. 11). In comparison, Remdesivir had a calculated $\mathrm{IC}_{50}$ of $8 \mu \mathrm{M}$. Thus, halofuginone showed a $\sim 1,000$-fold more potent inhibition of infection as compared to Remdesivir in this experimental setup (Fig. 4c-d). Similarly, halofuginone was 100-fold more potent compared to chloroquine $\left(\mathrm{IC}_{50} 1.9 \mu \mathrm{M}\right)$ (Extended data Fig. 12). Moreover, halofuginone treatment reduced SARS-CoV-2 spike intracellular mRNA levels more than 20,000-fold with an $\mathrm{IC}_{50}$ of $34.9 \mathrm{nM}$ (Fig. 4e).

We next examined if the impact of halofuginone on viral replication was dependent on PRS inhibition. Halofuginone competes with proline for the PRS active site ${ }^{15}$. Addition of excess proline to the media $(4 \mathrm{mM})$ of SARS-CoV-2 infected Vero E6 cells increased the $\mathrm{IC}_{50}$ of halofuginone from $12.5 \mathrm{nM}$ to $210 \mathrm{nM}$ (Fig 4f). Commercial halofuginone is a racemate of two, $2 S, 3 R-(-)$ and $2 R, 3 S-(+)$ enantiomers ${ }^{30}$. Hence, we evaluated if viral inhibition was due to ontarget effects of the PRS targeting $2 R, 3 S-(+)$ enantiomer (Fig. $4 \mathrm{~g})^{30}$. The $2 R, 3 S-(+)$ inhibited SARS-CoV-2 infectivity with an $\mathrm{IC}_{50}$ of $12 \mathrm{nM}$ compare to an $\mathrm{IC}_{50}$ of $28 \mathrm{nM}$ for the racemic mixture. No inhibition was observed when using the non-targeting $2 S, 3 R-(-)$ (Fig. 4g). Analogous, only the active $2 R, 3 S-(+)$ enantiomer inhibited HS production and authentic SARS-CoV-2 spike RBD protein binding (Extended data Fig. 13-14).

Like halofuginone, other PRS inhibitors ProSA and halofuginol inhibited authentic SARSCoV-2 infection of Vero E6 cells, although at significantly higher doses (Fig. 4h). The negative control compound, MAZ1310, did not (Fig. 4h). These results demonstrate that inhibition of PRS activity suppresses SARS-CoV-2 infection (Fig. 4h). Inhibitors of the ISR, as well as inhibitors of elF2-alpha kinases PERK and GCN2 were unable to attenuate the halofuginone-mediated inhibition of SARS-CoV-2 infectivity (Fig. 4i). Hence, the observed antiviral effect is not dependent on ISR activation, consistent with the effects seen on the binding of recombinant RBD spike 
protein and mAb 10E4 binding (Fig. 3c-d). ${ }^{31,32}$ Upon cell entry, SARS-CoV-2 genomic RNA is translated into two large polyproteins, pp1a (>400 kDa) and pp1ab (>700 kDa) that undergo proteolytic processing into 11 or 16 non-structural proteins, respectively, many of which are required for RNA replication. Both pp1a $(R 1 A)$ and pp1ab (R1AB), and to a lesser degree spike protein, have high proline contents comparable to collagens (Fig. 4j \& Extended data Fig. 15;

Extended data table 1). To probe the importance of the viral protein proline content versus general mRNA translation inhibition we evaluated two other AARS inhibitors, borrelidin and serylsulfamoyl adenosine (SerSA) that target the threonyl- and seryl-tRNA-synthetase, respectively. Niether of these AARS inhibitors were able to significantly attenuate viral replication (Fig. 4k) or Spike protein binding (Extended data Fig. 16), suggesting that proline translation is the Achilles heel for SARS-CoV-2 replication and possible other RNA viruses ${ }^{33,34}$.

\section{DISCUSSION}

We show that halofuginone is a potent inhibitor of SARS-CoV-2 infection in numerous cell types, including primary human bronchial epithelial cells. This small molecule decreases HSdependent spike protein binding, SARS-CoV-2 pseudovirus infection, and infection by authentic SARS-CoV-2. Interestingly, it also inhibits authentic SARS-CoV-2 infection post-entry by an HSindependent mechanism. Mechanistically, halofuginone suppresses SARS-CoV-2 infection by inhibiting the PRS, which could suppress the translation of long proline-rich host attachment factors, particularly HSPGs, and SARS-CoV-2 polyproteins pp1a and pp1ab that encode proteins required for viral replication. Thus, halofuginone is a potent host-targeting antiviral with dual inhibitory activity against SARS-CoV-2.

There is a desperate need for a potent, orally bioavailable antiviral for the treatment of COVID-19 that can be administered early in the disease to prevent hospitalization and the development of severe pulmonary disease. Here, we demonstrate that halofuginone is a potent inhibitor of SARS-CoV-2 infection with IC50 values in the low nanomolar range, in multiple in vitro 
models of infection. Halofuginone is orally bioavailable and reached an average $C_{\max }$ of $0.54 \mathrm{ng} / \mathrm{ml}$ $(\sim 1.3 \mathrm{nM})$ or $3.09 \mathrm{ng} / \mathrm{ml}(\sim 7.4 \mathrm{nM})$ after a single administration of $0.5 \mathrm{mg}$ or $3.5 \mathrm{mg}$ doses in a phase I clinical trial ${ }^{35}$. The long half-life $(\sim 30 \mathrm{~h})$ leads to accumulation of halofuginone, with twoto three-fold higher exposure by day 15 of dosing ${ }^{35}$. Halofuginone widely distributed in tissues after administration in mice with the highest tissue concentrations in the lung and kidney ${ }^{36}$. Expressed as area under the curve, halofuginone exposure was more than 87 -fold higher in the lung compared to plasma after a single intravenous injection in mice ${ }^{36}$. This suggests that although it may be difficult to obtain halofuginone plasma levels significantly above the $\mathrm{IC}_{50}$ values determined in this study, doses tested in phase I trials may be sufficient to achieve significant anti-SARS-CoV-2 activity in the lung and other organs infected by SARS-CoV-2 $2^{37-40}$.

Excessive inflammation can contribute to inflammatory organ injury during severe COVID19. There are multiple anti-inflammatory agents under evaluation for the treatment of COVID-19 and low dose dexamethasone recently demonstrated lower 28-day mortality in individuals receiving respiratory support ${ }^{41}$. In addition to the antiviral activity against SARS-CoV-2 that is described here, halofuginone has anti-inflammatory and anti-fibrotic activity that may provide yet another additive benefit to individuals with COVID-19 pneumonia ${ }^{1,2,15,42-44}$.

We demonstrate that other PRS inhibitors, ProSA and halofuginol, also inhibit infection by authentic SARS-CoV-2. Our data suggests that PRS inhibition may be particularly effective at suppressing the production of SARS-CoV-2 polyproteins pp1a and pp1ab that are long and proline-rich. Other positive-sense ssRNA viruses produce long polyproteins that may be similarly sensitive to PRS or other AARS inhibitors. Consistent with this idea, halofuginone demonstrates antiviral activity against future deleterious coronaviruses and other positive ssRNA viruses, including Chikungunya virus, Dengue virus, Zika virus, and Sindbis virus (Extended data Fig. 17; Extended data table 1$)^{45}$.

In addition to halofuginone, other PRS inhibitors are being evaluated in humans, including DWN12088 that is in phase I clinical trials in Australia and anticipated to be used for the treatment 
of interstitial pulmonary fibrosis. Thus, evaluation of PRS inhibitors, halofuginone analogs, and other AARS inhibitors may lead to the identification of additional broad-spectrum antiviral agents. Viruses require host cell resources for replication and thus can be inhibited by therapeutics that target essential host factors. This approach could provide broad activity against diverse viruses while decreasing the risk of emerging viral resistance as the therapy is not directed against a specific virally encoded product. On the other hand, targeting host factors can lead to cytostatic and cytotoxicity, and we did observe the reported anti-proliferative effect of halofuginone in our assays (Extended data Fig. 17) ${ }^{2}$. Although inhibition of global translation could lead to excessive toxicity, inhibiting AARSs could provide a more precise way of inhibiting viral proteins and thus limit toxicity. It appears that normal cells are relatively tolerant of decreased AARS levels with minimal effects on global translation. Individuals who are heterozygous carriers for recessive inactivating AARS mutations linked to hypomyelinating leukodystrophy do not display disease phenotypes ${ }^{46}$.

In conclusion, we identified halofuginone as an antiviral with potent inhibitory activity against SARS-CoV-2 infection in multiple human cell types. We show that halofuginone reduces both HS and HSPG biosynthesis, that are required for viral adhesion. In addition, we find that the inhibitory capacity of halofuginone on SARS-CoV-2 infection is not limited to HS reduction, but also shows potent inhibition in the viral replication stage. Mechanistically, our data suggests that these effects are caused by PRS inhibition and its effect on production of proline-rich proteins and not dependent on ISR activation. This observation is in agreement with previous studies reporting that coronaviruses overcome the inhibitory effects of elF2 $\alpha$ phosphorylation on viral mRNA translation ${ }^{31,32}$. Furthermore, many viruses, including coronaviruses (SARS1 and SARS2), shutdown host translation using nonstructural protein 1 (Nsp1) and activate elF2 $\alpha$ associated PERK dependent stress responses that benefit the virus ${ }^{47}$. Halofuginone may prevent SARSCoV-2 from circumventing these mechanisms of host protein translational shutdown. 
Halofuginone oral administration has been evaluated in a phase I clinical trial in humans and based on pharmacological studies in mice is distributed to SARS-CoV-2 target organs, including the lung. In conclusion, we show that halofuginone is a potent inhibitor of SARS-CoV-2 infection which emphasizes its potential as an effective treatment for COVID-19 in the clinic. Beyond its antiviral activity, halofuginone has potent anti-inflammatory and anti-fibrotic properties that may provide additive benefit in the treatment of COVID-19 pneumonia. Based on this in vitro preclinical data we believe halofuginone could be an effective antiviral and antifibrotic agent for the treatment of individuals with COVID-19. 


\section{REFERENCES AND NOTES}

1 Pines, M. \& Spector, I. Halofuginone - the multifaceted molecule. Molecules 20, 573-594, doi:10.3390/molecules20010573 (2015).

2 Luo, Y., Xie, X., Luo, D., Wang, Y. \& Gao, Y. The role of halofuginone in fibrosis: more to be explored? J Leukoc Biol 102, 1333-1345, doi:10.1189/jlb.3RU0417-148RR (2017).

3 Clausen, T. M. et al. SARS-CoV-2 Infection Depends on Cellular Heparan Sulfate and ACE2. Cell, doi:https:// doi.org/10.1016/j.cell.2020.09.033 (2020).

4 Beigel, J. H. et al. Remdesivir for the Treatment of Covid-19 - Preliminary Report. N Engl J Med, doi:10.1056/NEJMoa2007764 (2020).

5 Johns Hopkins University and Medicine, Johns Hopkins Coronavirus Resource Centre. https://coronavirus.jhu.edu (2020).

6 Food and Drug Administration. https://www.fda.gov/news-events/pressannouncements/coronavirus-covid-19-update-fda-issues-emergency-use-authorizationpotential-covid-19-treatment (2020).

7 Gillenwater, S., Rahaghi, F. \& Hadeh, A. Remdesivir for the Treatment of Covid-19 Preliminary Report. N Engl J Med 383, doi:10.1056/NEJMc2022236 (2020).

8 McMahon, J. H., Udy, A. \& Peleg, A. Y. Remdesivir for the Treatment of Covid-19 Preliminary Report. N Engl J Med 383, doi:10.1056/NEJMc2022236 (2020).

9 Wang, Y. et al. Remdesivir in adults with severe COVID-19: a randomised, double-blind, placebo-controlled, multicentre trial. Lancet 395, 1569-1578, doi:10.1016/S01406736(20)31022-9 (2020).

10 Spinner, C. D. et al. Effect of Remdesivir vs Standard Care on Clinical Status at 11 Days in Patients With Moderate COVID-19: A Randomized Clinical Trial. JAMA, doi:10.1001/jama.2020.16349 (2020).

11 McCreary, E. K. \& Angus, D. C. Efficacy of Remdesivir in COVID-19. JAMA, doi:10.1001/jama.2020.16337 (2020). 
12 Thorp, H. H. A dangerous rush for vaccines. Science 369, 885, doi:10.1126/science.abe3147 (2020).

13 Mycroft-West, C. J. et al. Heparin inhibits cellular invasion by SARS-CoV-2: structural dependence of the interaction of the surface protein (spike) S1 receptor binding domain with heparin. bioRxiv, 2020.2004.2028.066761, doi:10.1101/2020.04.28.066761 (2020).

14 Hoffmann, M. et al. SARS-CoV-2 Cell Entry Depends on ACE2 and TMPRSS2 and Is Blocked by a Clinically Proven Protease Inhibitor. Cell 181, 271-280 e278, doi:10.1016/j.cell.2020.02.052 (2020).

15 Keller, T. L. et al. Halofuginone and other febrifugine derivatives inhibit prolyl-tRNA synthetase. Nat Chem Biol 8, 311-317, doi:10.1038/nchembio.790 (2012).

16 Cribbs, A. et al. Inhibition of histone H3K27 demethylases selectively modulates inflammatory phenotypes of natural killer cells. J Biol Chem 293, 2422-2437, doi:10.1074/jbc.RA117.000698 (2018).

17 Cribbs, A. P. et al. Histone H3K27me3 demethylases regulate human Th17 cell development and effector functions by impacting on metabolism. Proc Natl Acad Sci U S A 117, 6056-6066, doi:10.1073/pnas.1919893117 (2020).

18 Cottone, L. et al. Inhibition of histone H3K27 demethylases inactivates brachyury (TBXT) and promotes chordoma cell death. Cancer Res, doi:10.1158/0008-5472.CAN-20-1387 (2020).

19 Zhou, H., Sun, L., Yang, X. L. \& Schimmel, P. ATP-directed capture of bioactive herbalbased medicine on human tRNA synthetase. Nature 494, 121-124, doi:10.1038/nature11774 (2013).

20 Blight, K. J., McKeating, J. A. \& Rice, C. M. Highly permissive cell lines for subgenomic and genomic hepatitis C virus RNA replication. J Virol 76, 13001-13014, doi:10.1128/jvi.76.24.13001-13014.2002 (2002). 
21 Pessentheiner, A. R., Ducasa, G. M. \& Gordts, P. Proteoglycans in Obesity-Associated Metabolic Dysfunction and Meta-Inflammation. Front Immunol 11, 769, doi:10.3389/fimmu.2020.00769 (2020).

22 David, G., Bai, X. M., Van der Schueren, B., Cassiman, J. J. \& Van den Berghe, H. Developmental changes in heparan sulfate expression: in situ detection with mAbs. J Cell Biol 119, 961-975, doi:10.1083/jcb.119.4.961 (1992).

23 Zhou, Y. et al. Metascape provides a biologist-oriented resource for the analysis of systems-level datasets. Nat Commun 10, 1523, doi:10.1038/s41467-019-09234-6 (2019).

24 Francklyn, C. S. \& Mullen, P. Progress and challenges in aminoacyl-tRNA synthetasebased therapeutics. J Biol Chem 294, 5365-5385, doi:10.1074/jbc.REV118.002956 (2019).

25 Adachi, R. et al. Discovery of a novel prolyl-tRNA synthetase inhibitor and elucidation of its binding mode to the ATP site in complex with I-proline. Biochem Biophys Res Commun 488, 393-399, doi:10.1016/j.bbrc.2017.05.064 (2017).

26 Costa-Mattioli, M. \& Walter, P. The integrated stress response: From mechanism to disease. Science 368, doi:10.1126/science.aat5314 (2020).

27 Fusakio, M. E. et al. Transcription factor ATF4 directs basal and stress-induced gene expression in the unfolded protein response and cholesterol metabolism in the liver. Mol Biol Cell 27, 1536-1551, doi:10.1091/mbc.E16-01-0039 (2016).

28 Kamtekar, S. et al. The structural basis of cysteine aminoacylation of tRNAPro by prolyltRNA synthetases. Proc Natl Acad Sci $U S S$ A 100, 1673-1678, doi:10.1073/pnas.0437911100 (2003).

29 Nakamura, A. et al. Inhibition of GCN2 sensitizes ASNS-low cancer cells to asparaginase by disrupting the amino acid response. Proc Natl Acad Sci U S A 115, E7776-E7785, doi:10.1073/pnas.1805523115 (2018). 
30 Jain, V. et al. Structure of Prolyl-tRNA Synthetase-Halofuginone Complex Provides Basis for Development of Drugs against Malaria and Toxoplasmosis. Structure 23, 819-829, doi:10.1016/j.str.2015.02.011 (2015).

31 Krahling, V., Stein, D. A., Spiegel, M., Weber, F. \& Muhlberger, E. Severe acute respiratory syndrome coronavirus triggers apoptosis via protein kinase $\mathrm{R}$ but is resistant to its antiviral activity. J Virol 83, 2298-2309, doi:10.1128/JVI.01245-08 (2009).

32 Nakagawa, K., Lokugamage, K. G. \& Makino, S. Viral and Cellular mRNA Translation in Coronavirus-Infected Cells. Adv Virus Res 96, 165-192, doi:10.1016/bs.aivir.2016.08.001 (2016).

33 Huter, P. et al. Structural Basis for Polyproline-Mediated Ribosome Stalling and Rescue by the Translation Elongation Factor EF-P. Mol Cell 68, 515-527 e516, doi:10.1016/j.molcel.2017.10.014 (2017).

34 Pavlov, M. Y. et al. Slow peptide bond formation by proline and other $\mathrm{N}$-alkylamino acids in translation. Proc Natl Acad Sci U S A 106, 50-54, doi:10.1073/pnas.0809211106 (2009).

35 de Jonge, M. J. et al. Phase I and pharmacokinetic study of halofuginone, an oral quinazolinone derivative in patients with advanced solid tumours. Eur J Cancer 42, 17681774, doi:10.1016/j.ejca.2005.12.027 (2006).

36 Stecklair, K. P. et al. Pharmacokinetics and tissue distribution of halofuginone (NSC 713205) in CD2F1 mice and Fischer 344 rats. Cancer Chemother Pharmacol 48, 375-382, doi:10.1007/s002800100367 (2001).

37 Ackermann, M. et al. Pulmonary Vascular Endothelialitis, Thrombosis, and Angiogenesis in Covid-19. N Engl J Med 383, 120-128, doi:10.1056/NEJMoa2015432 (2020).

38 Chan, J. F. et al. Simulation of the clinical and pathological manifestations of Coronavirus Disease 2019 (COVID-19) in golden Syrian hamster model: implications for disease pathogenesis and transmissibility. Clin Infect Dis, doi:10.1093/cid/ciaa325 (2020). 
39 Gupta, A. et al. Extrapulmonary manifestations of COVID-19. Nat Med 26, 1017-1032, doi:10.1038/s41591-020-0968-3 (2020).

40 Puelles, V. G. et al. Multiorgan and Renal Tropism of SARS-CoV-2. N Engl J Med 383, 590-592, doi:10.1056/NEJMc2011400 (2020).

41 Group, R. C. et al. Dexamethasone in Hospitalized Patients with Covid-19 - Preliminary Report. N Engl J Med, doi:10.1056/NEJMoa2021436 (2020).

42 Battu, S. et al. Amino acid starvation sensing dampens IL-1beta production by activating riboclustering and autophagy. PLoS Bio/ 16, e2005317, doi:10.1371/journal.pbio.2005317 (2018).

43 Luo, L. et al. Halofuginone attenuates intervertebral discs degeneration by suppressing collagen I production and inactivating TGFbeta and NF-small ka, CyrillicB pathway. Biomed Pharmacother 101, 745-753, doi:10.1016/j.biopha.2018.01.100 (2018).

44 Sundrud, M. S. et al. Halofuginone inhibits TH17 cell differentiation by activating the amino acid starvation response. Science 324, 1334-1338, doi:10.1126/science.1172638 (2009).

45 Hwang, J., Jiang, A. \& Fikrig, E. A potent prolyl tRNA synthetase inhibitor antagonizes Chikungunya and Dengue viruses. Antiviral Res 161, 163-168, doi:10.1016/j.antiviral.2018.11.017 (2019).

46 Kwon, N. H., Fox, P. L. \& Kim, S. Aminoacyl-tRNA synthetases as therapeutic targets. Nat Rev Drug Discov 18, 629-650, doi:10.1038/s41573-019-0026-3 (2019).

47 Thoms, M. et al. Structural basis for translational shutdown and immune evasion by the Nsp1 protein of SARS-CoV-2. Science, doi:10.1126/science.abc8665 (2020).

48 Son, J. et al. Conformational changes in human prolyl-tRNA synthetase upon binding of the substrates proline and ATP and the inhibitor halofuginone. Acta Crystallogr D Biol Crystallogr 69, 2136-2145, doi:10.1107/S0907444913020556 (2013). 
bioRxiv preprint doi: https://doi.org/10.1101/2021.03.22.436522; this version posted March 26, 2021. The copyright holder for this preprint (which was not certified by peer review) is the author/funder. All rights reserved. No reuse allowed without permission.

49 Song, Y. et al. Double mimicry evades tRNA synthetase editing by toxic vegetablesourced non-proteinogenic amino acid. Nat Commun 8, 2281, doi:10.1038/s41467-01702201-z (2017). 


\section{ACKNOWLEDGMENTS}

We thank John Christianson, University of Oxford, and Matthew Frieman at the University of Maryland for discussions and helpful comments, and Alexandra Efstrathiou, University of Oxford, for technical assistance.

Funding: This work was supported by Foundation Leducq 16CVD01 to P.L.S.M.G., U.O., R.M.E. and S.T., a UCSD Innovation Grant 13991 to P.L.S.M.G., a RAPID grant 2031989 from the National Science Foundation and Project 3 of NIH P01 HL131474 to J.D.E.; The Alfred Benzon Foundation to T.M.C; NIH grant 5T32GM127235-02 to J.K.C.C.; NIH fellowship support K12HL141956 to R.J.W.; The Carlsberg Foundation Fellowship to K.V.G.; a Career Award for Medical Scientists from the Burroughs Wellcome Fund to A.F.C. NIH Grant R01 HL124209, the American Asthma Foundation, and the BSF 2017176 to B.A.C.; COVID19 seed funding from the Huck Institutes of the Life Sciences and Penn State start-up funds to J.J.; R.M. NIH 1R21Al132981-01, the Bill and Melinda Gates Foundation OPP1086203 and Cancer Research UK and Arthritis Research UK 20522 grants to U.O.

\section{Competing interests:}

S.T. is a founding member of Oxitope, Inc, Covicept Therapeutics and Kleanthi Diagnostics.

S.T. is a consultant for lonis Pharmaceuticals. PL.S.M.G. is a is a founding member of Covicept Therapeutics. J.D.E. is a co-founder of TEGA Therapeutics and Covicept Therapeutics. J.D.E. and The Regents of the University of California have licensed a University invention to and have an equity interest in TEGA Therapeutics and Covicept Therapeutics. The terms of this arrangement have been reviewed and approved by the University of California, San Diego in accordance with its conflict of interest policies. NIH R01 Al146779 and a Massachusetts Consortium on Pathogenesis Readiness (MassCPR) grant to A.G.S.; training grants: NIGMS T32 GM007753 for B.M.H. and T.M.C; T32 AI007245 for J.F. R.M. is a scientific advisory board (SAB) member and equity holder of Regenacy Pharmaceuticals, ERX Pharmaceuticals,

Frequency Therapeutics. D.R.S, T.M.C., C.N., A.D., RJ.W., J.D.E., P.L.S.M.G. R.M., M.A.T. and 
N.C.P. are inventors on patent applications related to PRS inhibitors. The other authors declare that they have no competing interests.

Author contributions: P.L.S.M.G., A.F.C., D.R.S., T.M.C. and C.N. conceived the project; P.L.S.M.G., A.F.C., J.D.E., J.J., S.T., B.A.C., S.L., R.M., B.A.C., K.Z., U.O., D.R.S., T.M.C. R.J. W. and C.N designed and performed the experimental work.; A.D., A.P.C., A.E.C., A.F.G, J.K.C.K., A.N., S.A.M., G.J.G., C.B.S, C.J.N., E.R.G., J.E.D, C.J.N, E.R.G, E.M.K., R.N.M. and A.P. performed the experimental work.: X.Y., Y.P., J.F., B.H., T.M.C., S.K.C., M.A.T., N.C.P, A.G.S. J.A.M., T.A., C.B, M.D., R.M.E. and R.M. supplied reagents. P.L.S.M.G., A.F.C. D.R.S., T.M.C., J.K.C.K., J.D.E., U.O., R.M., J.A.M., M.D., R.M.E., A.P.C., M.P, C.J., B.A.C., K.V.G. and S.T. analyzed and discussed the data.; T.M.C., D.R.S., A.F.C. and P.L.S.M.G, wrote the manuscript. All authors discussed the experiments and results, read, and approved the manuscript.

\section{Materials \& Correspondence.}

Correspondence should be addressed to: Philip L.S.M. Gordts, Department of Medicine, University of California, La Jolla, CA 92093, USA; +1(858)246-0994, pgordts@health.ucsd.edu, Aaron F. Carlin, Department of Medicine, University of California, La Jolla, CA 92093, USA; +1(858)246-3261, acarlin@health.ucsd.edu 
Figure 1

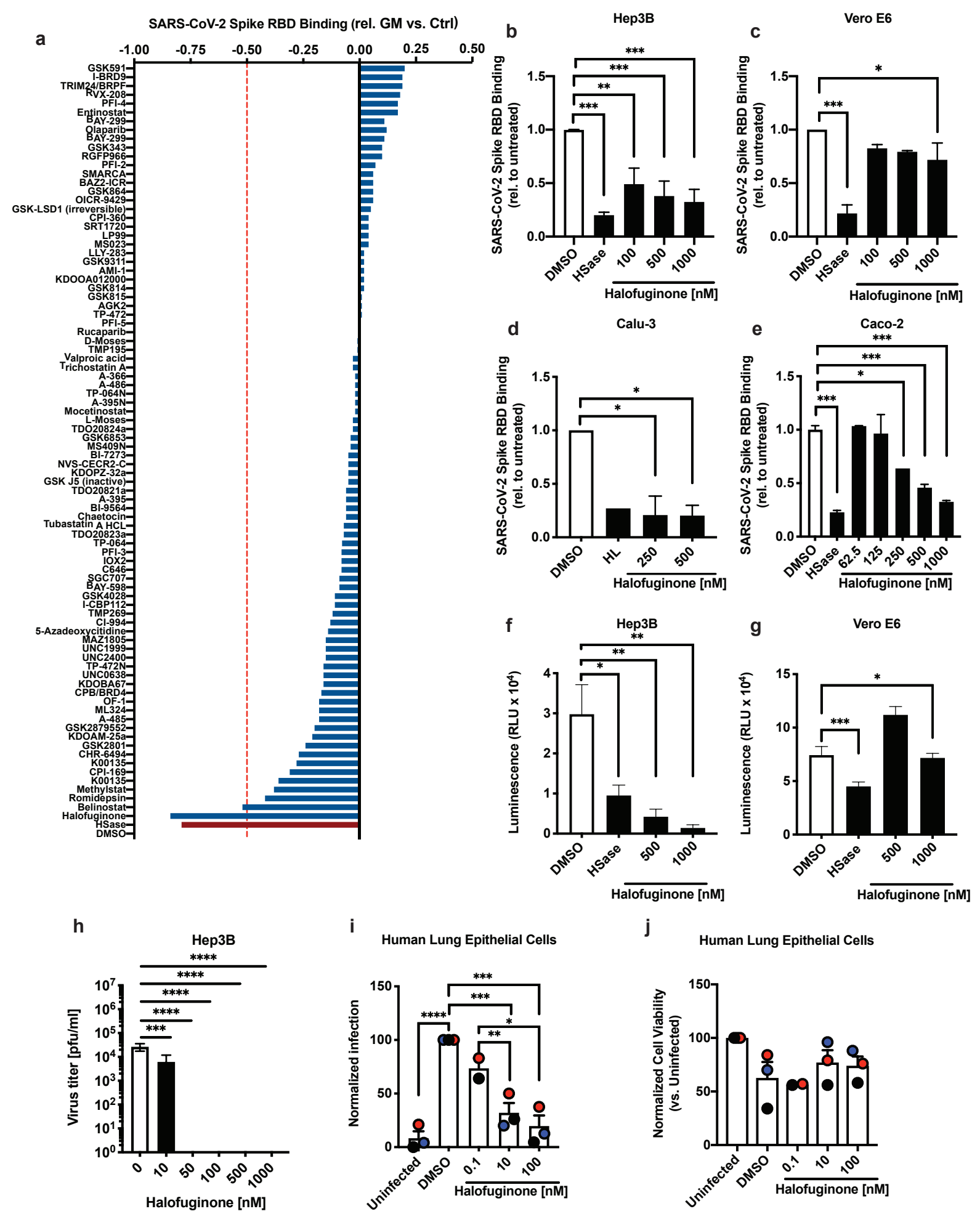

Figure 1. Screen of epigenetic and translational regulatory compounds identify 
Halofuginone as a potent inhibitor of SARS-CoV-2 Spike HS dependent cellular adhesion.

a, Hep3B cells were treated with a library of epigenetic and translational regulatory compounds or heparin lyase (HSase) as a positive control and tested for their interaction with recombinant SARS-CoV-2 RBD protein. b-e, Titration of halofuginone on Hep3B cells (b), Vero E6 (c), Calu-3 (d) and Cacu-2 (e) cells ( $n=2-4$ replicates per condition), and its effect on binding of recombinant SARS-CoV-2 RBD protein Heparin Lyase treatment is included as control for HS dependent adhesion. $\mathbf{f}-\mathbf{g}$, effect of halofuginone treatment on the infection of SARS-CoV-2 spike protein pseudotyped virus in Hep3B (f) and Vero E6 (g) cells ( $n=3-4$ replicates per condition). $\mathbf{h}$, Authentic SARS-CoV-2 virus infection of Hep3B cells treated with halofuginone. i, Infection of primary human bronchial epithelial cells, grown at an air-liquid interface, treated with halofuginone (Red and black colors represent the two different primary human bronchial epithelial cells isolates used). j, Relative cell viability of primary human bronchial epithelial cells. Data shown as mean \pm S.D. Statistics performed by 1-way ANOVA and uncorrected Fisher's LSD test (ns: $p>0.05,{ }^{*}: p$ $\left.\leq 0.05,{ }^{* *}: p \leq 0.01,{ }^{* * *}: p \leq 0.001\right)$. 
a

Figure 2
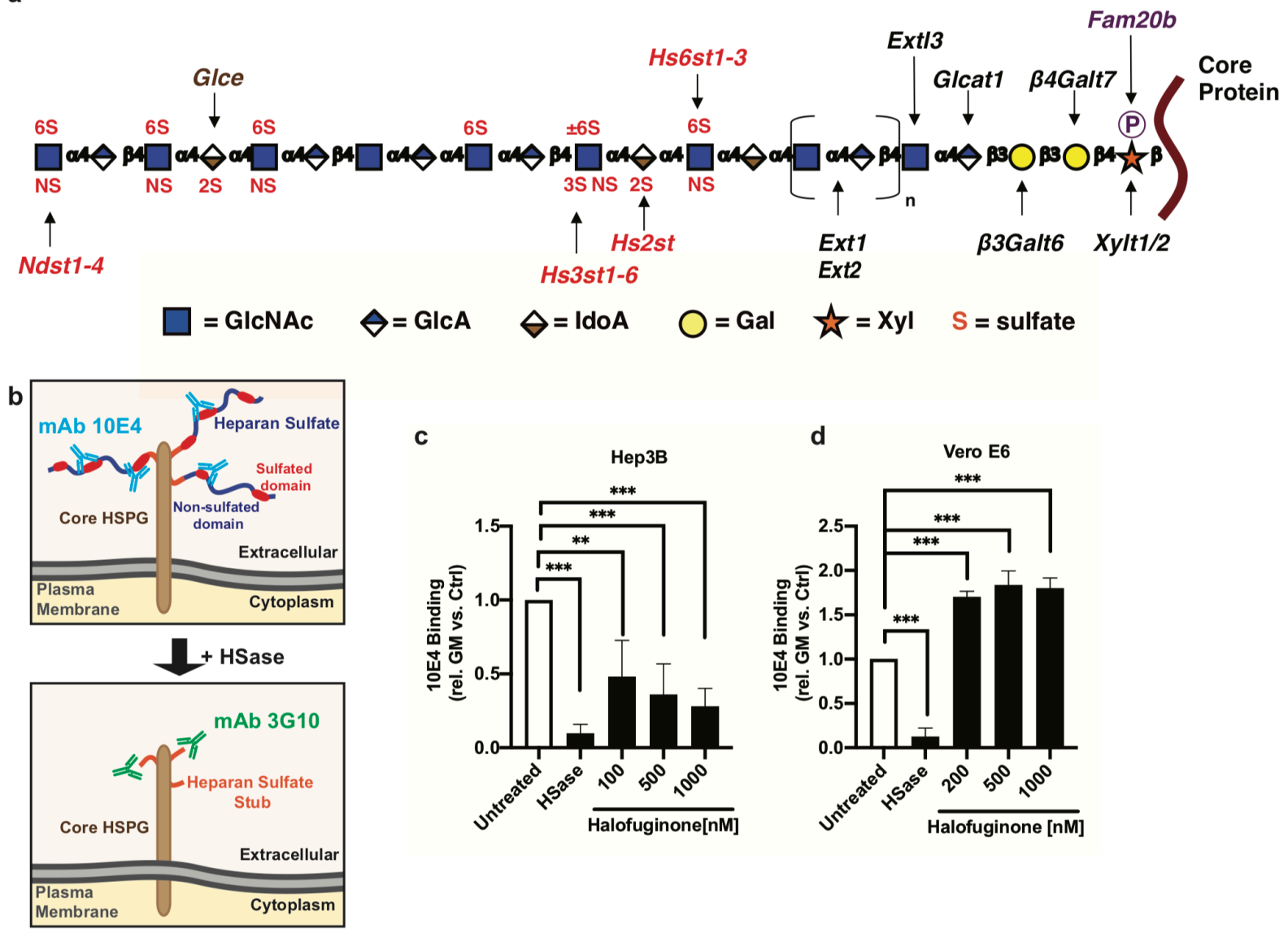

C
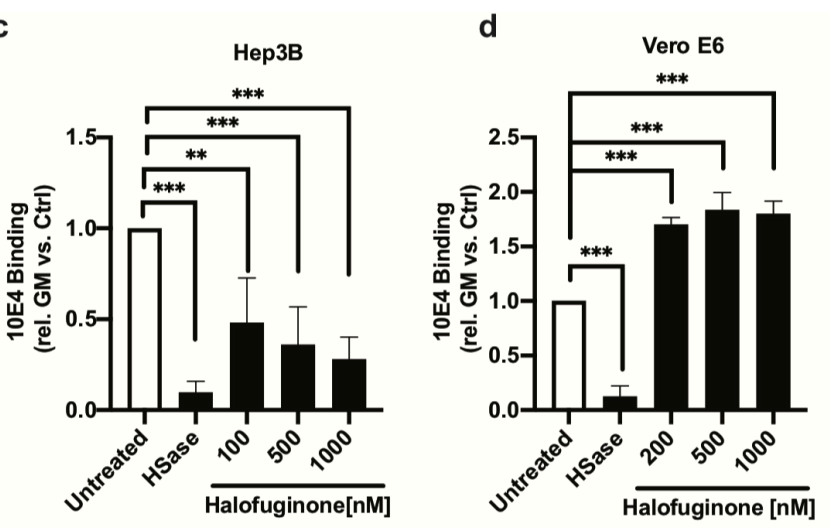

e
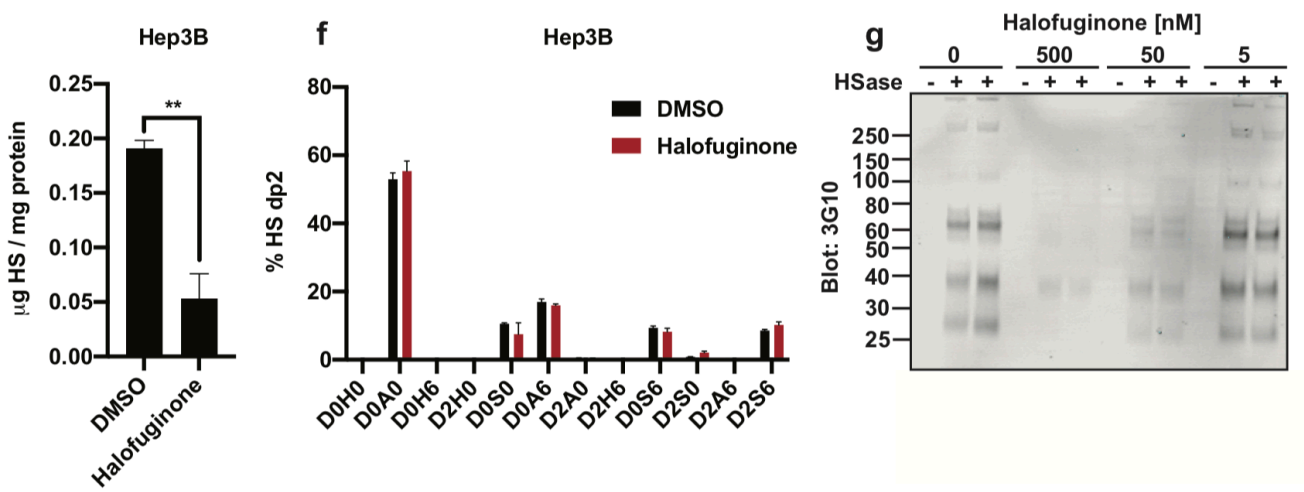

Figure 2. Halofuginone Inhibits Heparan Sulfate Biosynthesis. a, Schematic representation of HS biosynthesis. Genes required for priming and elongation are highlighted in black. Sulfotransferases and other modifying enzymes are highlighted in red. b, Schematic example of the interaction with the anti-HS 10E4 and 3G10 mAb's. 10E4 recognizes sulfated HS polymer, while $3 \mathrm{G} 10$ recognizes the number of HS attachments sites by interacting with the stub left after 
heparin lyase (HSase) treatment. c-d, titration of halofuginone on Hep3B cells (c), Vero E6 (d), and its effect on cellular staining with anti-HS 10E4 mAb ( $n=3$ per group). e, quantitative determination of HS content in Hep3B cells treated with halofuginone. Absolute HS content was determined by disaccharide analysis using LC-MS ( $n=3$ per group). f, Qualitative distribution of specific sulfation patterns in Hep3B cells treated with halofuginone, seen in the LC-MS analysis ( $n=3$ per group). g, Quantification of functional HS binding sites in Hep3B cells treated with halofuginone. Binding sites are quantified in SDS-PAGE using mAb $3 G 10$ that recognizes the linker tetrasaccharide bound to the HSPG core protein after hep lyase treatment. Data shown as mean \pm S.D. Statistics performed by unpaired $t$ test or 1-way ANOVA and uncorrected Fisher's LSD test (ns: $p>0.05,{ }^{*}: p \leq 0.05,{ }^{* *}: p \leq 0.01,{ }^{* * *}: p \leq 0.001,{ }^{* * * *}: p \leq 0.0001$ ). 
A

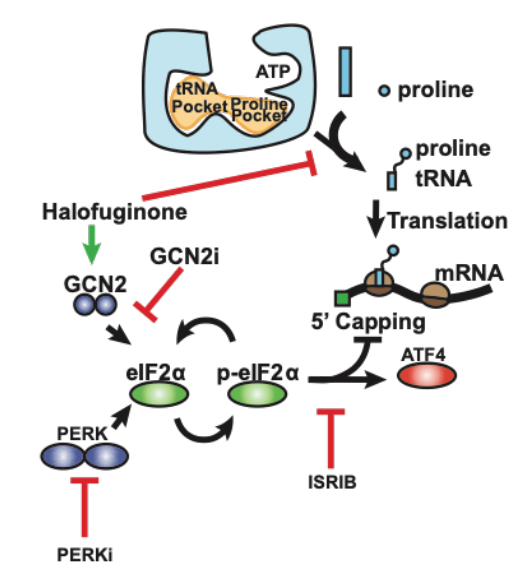

B

B Figure 3

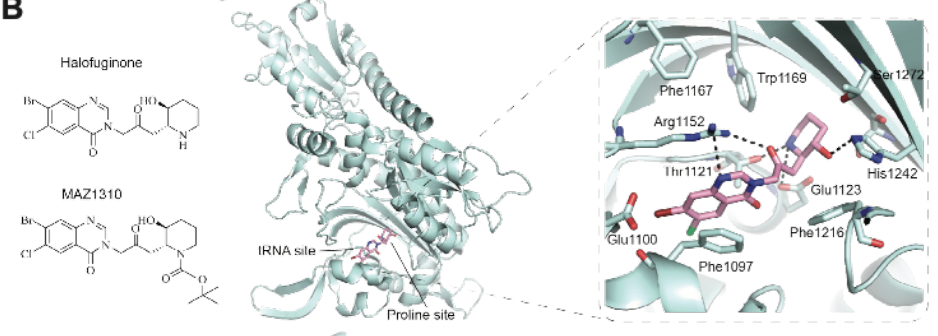

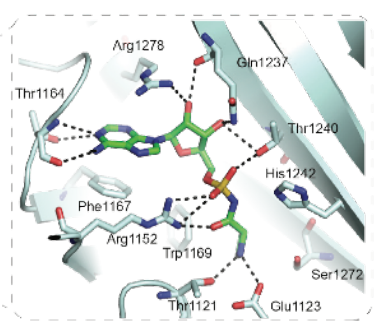

$\mathbf{F}$

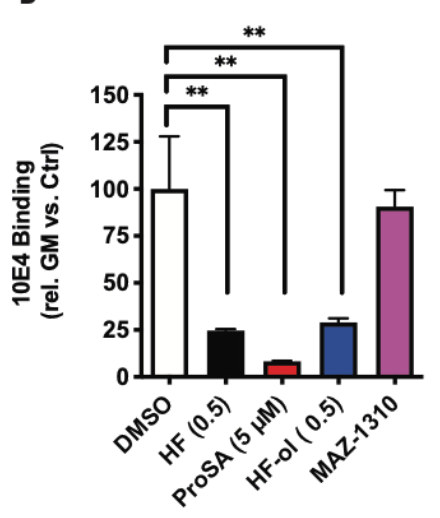

G

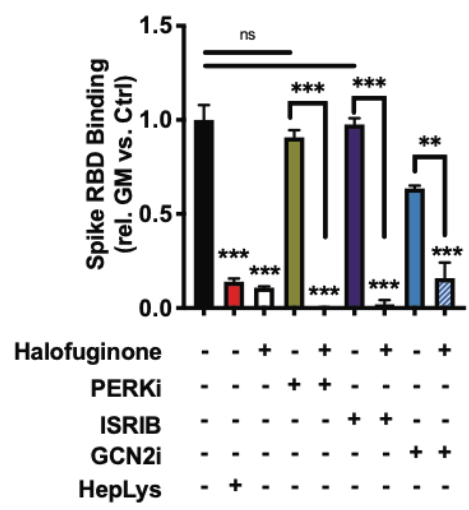

E

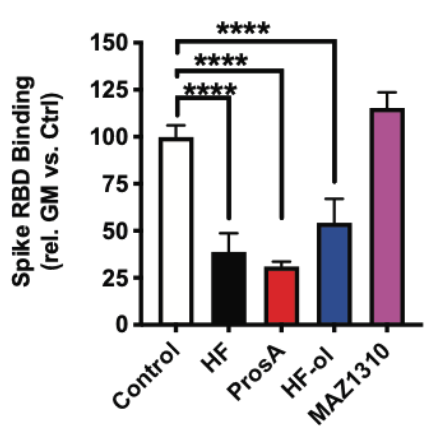

H

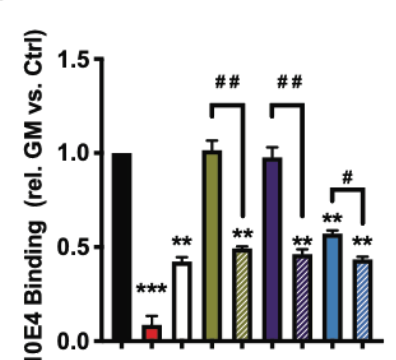

Halofuginone

PERKi

GCN2i - . . . - +

HepLys - + . - . -

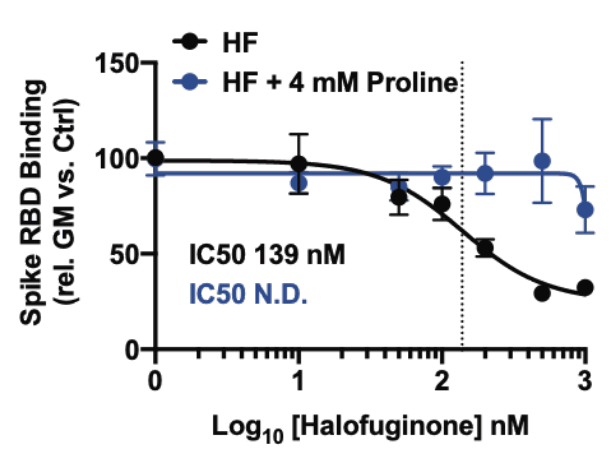

I

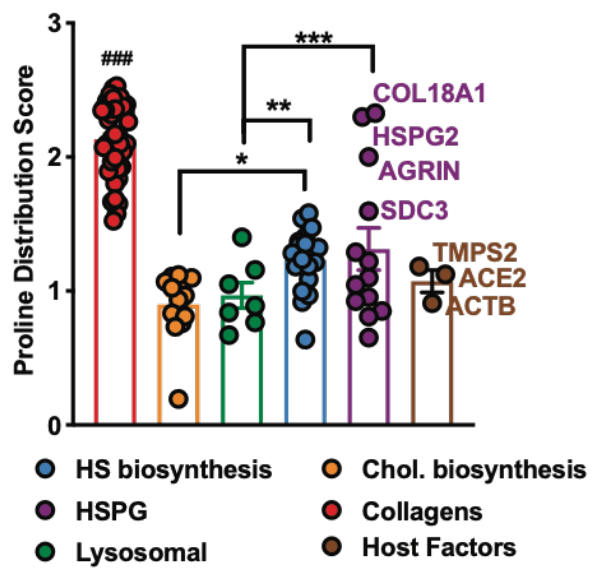

Figure 3. Halofuginone Inhibition of heparan sulfate presentation and Spike protein binding is not dependent of the integrated stress response. a, Chemical structure of halofuginone and negative control compound MAZ1310. Figure shows the interaction of 
halofuginone with the human prolyl-tRNA synthetase (PRS) active site, as resolved by X-ray crystallography (PDB: 4K88) ${ }^{48}$. b, Chemical structure of ProSA. Graphic shows the interaction of ProSA with human PRS (PDB: 5V58) ${ }^{49}$. c, Schematic representation of the mediators of the integrated stress response (ISR). c-d, Treatment of Hep3B cells with modulators of the PRS pathway at $0.5 \mu \mathrm{M}$ (ProSA at $5 \mu \mathrm{M}$ ) and its effect on (c) HS presentation as measured by anti-HS mAb 10E4 binding and (d) spike RBD binding (e) by flow cytometry. Binding is represented as relative to non-treated control. f, Treatment of Hep3B cells with modulators of the halofuginone with or without $4 \mathrm{mM}$ proline and its effect on spike RBD binding by flow cytometry. Binding is represented as relative to non-treated control. $\mathbf{g}$, Treatment of Hep3B cells with modulators of the ISR and its effect on HS presentation as measured by anti-HS mAb 10E4 binding in flow cytometry. $\mathbf{h}$, Treatment of Hep3B cells with modulators of the ISR and its effect on SARS-CoV2 recombinant $\mathrm{RBD}$ protein binding in flow cytometry. Binding is represented as relative to nontreated control. i, The distribution of proline distribution and density depicted as a proline distribution score for collagens, cholesterol biosynthetic proteins, lysosomal proteins, heparan sulfate (HS) biosynthetic proteins, heparan sulfate proteoglycans (HSPG) and viral host factor proteins ( ${ }^{\#} p \leq 0.01$ collagens vs. all other protein classes). Data shown as mean \pm S.D. Statistics performed by 1 -way ANOVA and uncorrected Fisher's LSD test (ns: $p>0.05,{ }^{*}: p \leq 0.05,{ }^{* *}: p \leq$ $\left.0.01,{ }^{* * *}: p \leq 0.001,{ }^{* * * *}: p \leq 0.0001 ;{ }^{\#}: p \leq 0.05,{ }^{\# \#}: p \leq 0.01\right)$. 


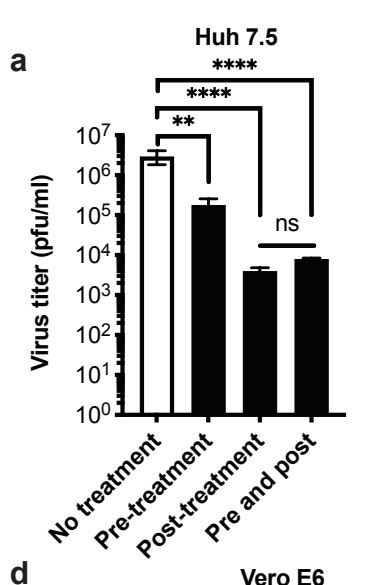

b

Huh 7.5

Figure 4

d
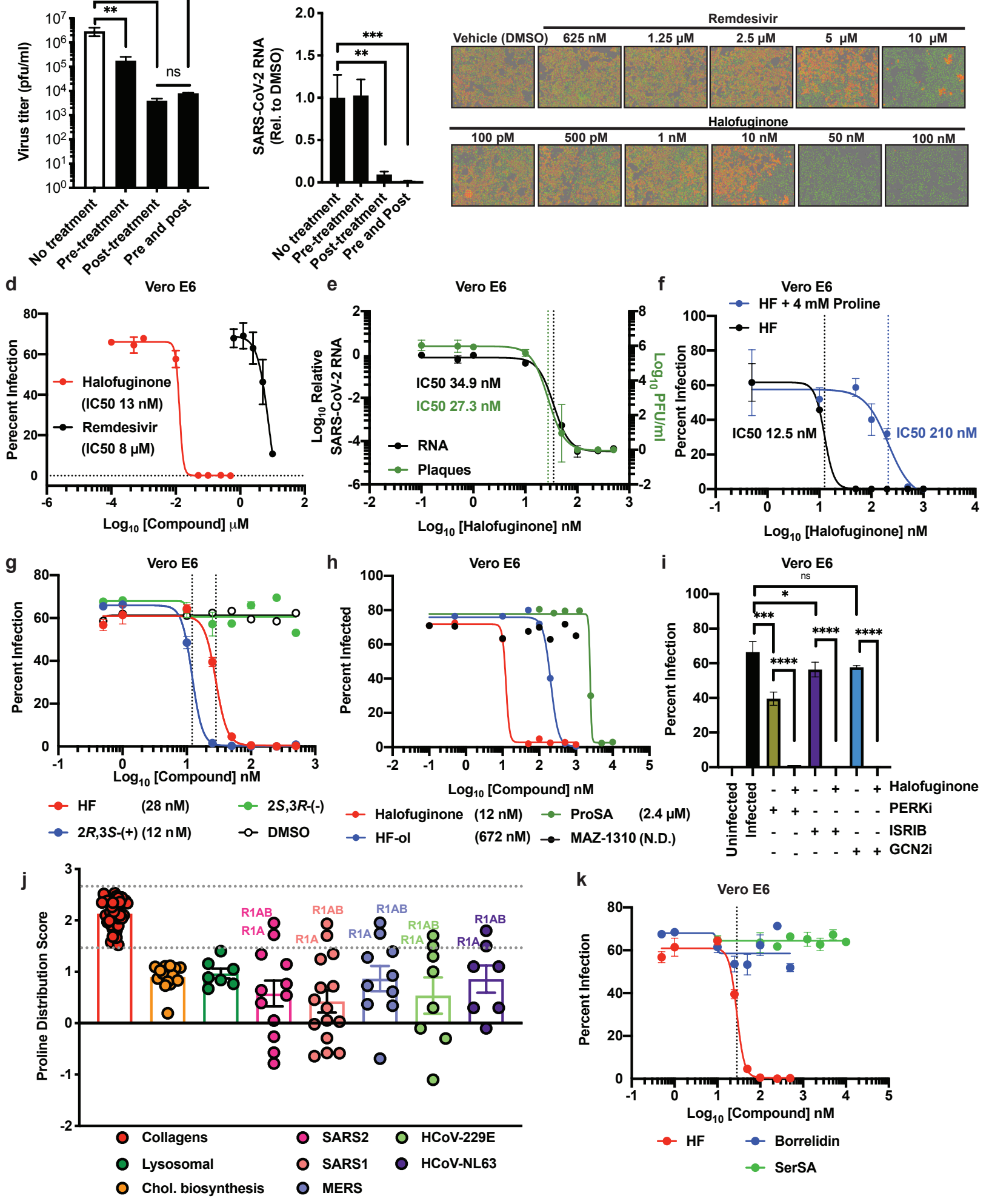

Figure 4. Halofuginone Inhibits infection and replication of authentic SARS-CoV-2. a-b, 
Authentic SARS-CoV-2 virus infection of Huh7.5 cells treated with Halofuginone. Huh7.5 cells treated with halofuginone pre- or post-infection, or both pre- and post-infection with authentic SARS-CoV-2 virus. Viral titers (a) and quantification of viral RNA (b) in the infected cells is shown. c, Immunofluorescent quantification of viral nucleocapsid (red) protein in Vero E6 cells treated with Halofuginone and Remdesivir and infected with authentic SARS-CoV-2 virus (nuclei = green). d, Authentic SARS-CoV-2 virus infection of Vero E6 cells treated with Halofuginone and Remdesivir as measured in flow cytometry. e, Quantification of plaque formation and viral RNA in Vero E6 cells treated with Halofuginone and Remdesivir and infected with authentic SARS-CoV2 virus. $\mathbf{f}$, Rescue experiment of the effect of halofuginone treatment on SARS-CoV-2 infection using excess proline. g, Treatment of Vero E6 cells with halofuginone and enantiomers and their effect on SARS-CoV-2 infection. $\mathbf{h}$, Treatment of Vero E6 cells with modulators of the PRS pathway and their effect on SARS-CoV-2 infection. i, Treatment of Vero E6 cells with modulators of the ISR pathway and their effect on SARS-CoV-2 infection. $\mathbf{j}$, The distribution of proline distribution and density depicted as a proline distribution score for collagens, cholesterol biosynthetic proteins, lysosomal proteins and viral SARS-CoV-2 (SARS2), SARS-CoV-1 (SARS1), MERS-CoV (MERS), HCoV-229E and HCoV-LN63 proteins $\left({ }^{\# \#} p \leq 0.01\right.$ collagens vs. all other protein classes). k, Treatment of Vero E6 cells with AARS inhibitors and their effect on SARS-CoV-2 infection. Data shown as mean \pm S.D. Statistics performed by 1 -way ANOVA and uncorrected Fisher's LSD test (ns: $p>0.05,{ }^{*}: p \leq 0.05,{ }^{* *}: p \leq 0.01,{ }^{* * *}: p \leq 0.001,{ }^{* * * *}: p \leq 0.0001$, $\#$ \# $p \leq 0.01$. 


\section{METHODS}

\section{Reagents}

SARS-CoV-2 (2019-nCoV) Spike Protein (RBD, His Tag) (Sino Biologicals, 40592-V08B), and proteins were biotinylated using EZ-Link ${ }^{\mathrm{TM}}$ Sulfo-NHS-Biotin, No-Weigh ${ }^{\mathrm{TM}}$ (Thermo Fisher Scientific, A39256). Heparin lyases were from IBEX pharmaceuticals, Heparinase I (IBEX, 60012), Heparinase II (IBEX, 60-018), Heparinase III (IBEX, 60-020). Protein production reagents included, Pierce ${ }^{\mathrm{TM}}$ Protein Concentrators PES Thermo Scientific ${ }^{\mathrm{TM}}$, Pierce ${ }^{\mathrm{TM}}$ Protein Concentrator PES (Thermo Fisher Scientific, 88517) and Zeba ${ }^{\text {TM }}$ Spin Desalting Columns, 40K MWCO, $0.5 \mathrm{~mL}$ (Thermo Fisher Scientific, 87766). ISR pathway inhibitors used are ISRIB (Sigma, SML0843), GSK2606414 (Sigma, 516535), and A-92 (Axon, 2720). Antibodies used were, anti-Spike antibody [1A9] (GeneTex, GTX632604), anti-Nucleocapsid antibody (GeneTex, GTX135357), Anti-HS (Clone F58-10E4) (Fisher Scientific, NC1183789), and 3G10 (BioLegend, 4355S). Secondary antibodies were, Anti-His-HRP (Genscript, A00612), Avidin-HRP

(Biolegend, 339902), and Streptavadin-Cy5 (Thermo Fisher, SA1011). Luciferase activity was monitored by Bright-Glo ${ }^{\mathrm{TM}}$ (Promega, E2610). All cell culture medias and PBS where from Gibco.

\section{Cell Culture}

Huh-7.5 cells ${ }^{1}$ were generously provided by Charles M. Rice (Rockefeller University, New York, NY). Hep3B, BHK-15 and Calu-3 cells were from ATCC and were grown in DMEM medium containing $10 \% \%$ fetal bovine serum, and $100 \mathrm{IU} / \mathrm{ml}$ of penicillin, $100 \mu \mathrm{g} / \mathrm{ml}$ of streptomycin sulfate and nonessential amino acids. BHK-15 were grown in Modified Eagle's supplemented with $10 \%$ fetal bovine serum and nonessential amino acids (Gibco, \#11140-050). Hep3B, A375 and Vero E6 cells were from ATCC. The Hep3B cells carrying mutations in HS biosynthetic enzymes was derived from the parent ATCC Hep3B stock, and have been described previously 2. All cells were supplemented with $10 \% \mathrm{FBS}, 100 \mathrm{IU} / \mathrm{ml}$ of penicillin and $100 \mu \mathrm{g} / \mathrm{ml}$ of 
streptomycin sulfate and grown under an atmosphere of 5\% CO2 and 95\% air. Cells were passaged before $80 \%$ confluence was reached and seeded as explained for the individual assays. Cell viability was measured using LDH leakage (Promega) or alamarBleu (Thermo Fisher).

\section{SARS-CoV-2 spike RBD protein production}

Recombinant SARS-CoV-2 RBD (GenBank: MN975262.1; amino acid residues 319-529) was cloned into pVRC vector containing a HRV 3C-cleavable C-terminal SBP-His $8 x$ tag was produced in ExpiCHO cells by transfection of $6 \times 10^{6}$ cells $/ \mathrm{ml}$ at $37^{\circ} \mathrm{C}$ with $0.8 \mu \mathrm{g} / \mathrm{ml}$ of plasmid DNA using the ExpiCHO expression system transfection kit in ExpiCHO Expression Medium (ThermoFisher). One day later the cells were fed with ExpiCho Feed, treated with ExpCho Enhancer, and then incubated at $32{ }^{\circ} \mathrm{C}$ for 11 days. The conditioned medium was mixed with cOmplete EDTA-free Protease Inhibitor (Roche). Recombinant protein was purified by chromatography on a $\mathrm{Ni}^{2+}$ Sepharose 6 Fast Flow column (1 ml, GE LifeSciences). Samples were loaded in ExpiCHO Expression Medium supplemented with $30 \mathrm{mM}$ imidazole, washed in a $20 \mathrm{mM}$ Tris-Cl buffer $(\mathrm{pH}$ 7.4) containing $30 \mathrm{mM}$ imidazole and $0.5 \mathrm{M} \mathrm{NaCl}$. Recombinant protein was eluted with buffer containing $0.5 \mathrm{M} \mathrm{NaCl}$ and $0.3 \mathrm{M}$ imidazole. The protein was further purified by size exclusion chromatography (HiLoad 16/60 Superdex 200, prep grade. GE LifeSciences) in 20 mM HEPES buffer ( $\mathrm{pH} 7.4$ ) containing $0.2 \mathrm{M} \mathrm{NaCl}$.

\section{SARS-CoV-2 Spike RBD Biotinylation}

For binding studies, recombinant SARS-CoV-2 Spike RBD protein (RBD, His Tag) was conjugated with EZ-Link ${ }^{\mathrm{TM}}$ Sulfo-NHS-Biotin (1:3 molar ratio; Thermo Fisher) in Dulbecco's PBS at room temperature for $30 \mathrm{~min}$. Glycine $(0.1 \mathrm{M})$ was added to quench the reaction and the buffer was exchanged for PBS using a Zeba spin column (Thermo Fisher). 


\section{Flow cytometry}

Cells at $50-70 \%$ confluence were treated with halofuginone or other inhibitors for $18-24 \mathrm{hrs}$. The cells were lifted with PBS containing $10 \mathrm{mM}$ EDTA (Gibco) and washed in PBS containing 0.1\% BSA. The cells were seeded into a 96 -well plate at $10^{5}$ cells per well. As a control for HSdependent spike protein adhesion, a portion of the cells were treated with HSase $\mathrm{mix}(2.5 \mathrm{mU} / \mathrm{ml}$ HSase I, 2.5 or $5 \mathrm{mU} / \mathrm{ml}$ HSase II, and $5 \mathrm{mU} / \mathrm{ml} \mathrm{HSase} \mathrm{III;} \mathrm{IBEX)} \mathrm{for} 30 \mathrm{~min}$ at $37^{\circ} \mathrm{C}$ in PBS containing $0.1 \% \mathrm{BSA}$. Cells were stained for $30 \mathrm{~min}$ at $4^{\circ} \mathrm{C}$ with biotinylated spike RBD protein $(20 \mu \mathrm{g} / \mathrm{ml})$ or $10 \mathrm{E} 4 \mathrm{mAB}$ in PBS containing $0.1 \% \mathrm{BSA}$. The cells were washed twice and then reacted for $30 \mathrm{~min}$ at $4^{\circ} \mathrm{C}$ with Streptavadin-Cy5 (Thermo Fisher; 1:1000 dilution) or anti-mouse IgG-Alexa488, respectively, in PBS containing 0.5\% BSA. The cells were washed twice and then analyzed using a FACSCalibur or a FACSCanto instrument (BD Bioscience). All experiments were done a minimum of three separate times in three technical replicates. Data analysis was performed using FlowJo software and statistical analyses were done in Prism 8 (GraphPad).

\section{HS and CS purification from cell surface of Hep3B cells}

Fresh Hep3B cells were was washed in PBS, lifted with trypsin, and centrifuged for 5 min a 500 $x \mathrm{~g}$. The trypsin supernatants were saved for GAG analysis, and the cell pellets were washed in complete media, followed by a PBS wash, and saved for BCA analysis (Pierce ${ }^{\mathrm{TM}}$ BCA Protein Assay Kit, Themo Fisher), according to vendor specifications. The trypsin-released GAGs were treated with $1 \mathrm{mg} / \mathrm{mL}$ Pronase (Streptomyces griseus, Sigma Aldrich) and 0.1\% Triton X-100 and $10 \mathrm{mM} \mathrm{CaCl}_{2}$, and incubated at $37^{\circ} \mathrm{C}$, overnight with shaking. The samples were centrifuged at $20,000 \times \mathrm{g}$ for $20 \mathrm{~min}$ and the supernatant was mixed 1:10 with equilibration buffer (50 mM sodium acetate, $0.2 \mathrm{M} \mathrm{NaCl}, 0.1 \%$ Triton $\mathrm{X}-100, \mathrm{pH} 6$ ) and loaded onto a DEAE Sephacel column (GE healthcare) equilibrated with equilibration buffer. The column was washed with wash buffer ( $50 \mathrm{mM}$ sodium acetate, $0.2 \mathrm{M} \mathrm{NaCl}, \mathrm{pH} 6.0)$ and bound GAGs were eluted with elution buffer ( $50 \mathrm{mM}$ sodium acetate, $2.0 \mathrm{M} \mathrm{NaCl}, \mathrm{pH} 6.0$ ). The eluate was desalted 
using PD-10 columns (GE Healthcare) and lyophilized until dry.. The GAG pellets were reconstituted in DNase buffer (50 mM Tris, $50 \mathrm{mM} \mathrm{NaCl}, 2.5 \mathrm{mM} \mathrm{MgCl}_{2}, 0.5 \mathrm{mM} \mathrm{CaCl}$, pH 8.0) with $20 \mathrm{kU} / \mathrm{mL}$ DNase I (Deoxyribonuclease I from Bovine Pancreas, Sigma Aldrich) and incubated for $2 \mathrm{hr}$ at $37^{\circ} \mathrm{C}$, shaking. Finally, the GAGs were purified over a DEAE column and precipitated by PD-10 chromatography ${ }^{3}$.

\section{HS and CS digestion and MS analysis}

For HS quantification and disaccharide analysis, purified GAGs were digested with a mixture of heparin lyases I-III (2 mU each) for $2 \mathrm{hr}$ at $37^{\circ} \mathrm{C}$ in lyase buffer (40 mM ammonium acetate and $3.3 \mathrm{mM}$ calcium acetate, $\mathrm{pH}$ 7.0). For CS quantification and disaccharide analysis, the GAGs were digested with $20 \mathrm{mU} / \mathrm{mL}$ Chondroitinase ABC (from Proteus vulgaris, Sigma Aldrich) and incubated for $2 \mathrm{hr}$ at $37^{\circ} \mathrm{C}$ in lyase buffer (50 mM Tris and $\left.50 \mathrm{mM} \mathrm{NaCl}, \mathrm{pH} 8.0\right)$. The reactions were dried in a centrifugal evaporator and tagged by reductive amination with $\left[{ }^{12} \mathrm{C}_{6}\right]$ aniline. The HS and CS samples were analyzed by liquid chromatography (LC) coupled to tandem mass spectrometry (MS/MS) and quantified by inclusion of $\left[{ }^{13} \mathrm{C}_{6}\right]$ aniline-tagged standard $\mathrm{HS}$ disaccharides (Sigma-Aldrich), as described (Lawrence et al., 2008). The samples were separated on a reverse phase column (TARGA C18, $150 \mathrm{~mm} \times 1.0 \mathrm{~mm}$ diameter, $5 \mu \mathrm{m}$ beads, Higgins Analytical, Inc.) using $5 \mathrm{mM}$ dibutylamine as an ion pairing agent (Sigma-Aldrich), and ions were monitored in negative mode. Separation was performed using the same gradient, capillary temperature, and spray voltage as described (Lawrence et al., 2008). The analysis was done on an LTQ Orbitrap Discovery electrospray ionization mass spectrometer (Thermo Scientific) equipped with an Ultimate 3000 quaternary HPLC pump (Dionex).

\section{Western Blot Analysis}

Cells were lysed using RIPA buffer, and protein was quantified using a BCA assay. Protein was analyzed by SDS-PAGE on 4-12\% Bis-Tris gradient gels (NuPage; Invitrogen) with an equal 
amount of protein loading. Proteins were visualized after transfer to Immobilon-FL PVDF membrane (Millipore). Membranes were blocked with Odyssey blocking buffer (LI-COR Biosciences) for $30 \mathrm{~min}$ and incubated overnight at $4^{\circ} \mathrm{C}$ with $3 \mathrm{G} 10$ antibodies. Mouse antibodies were incubated with secondary Odyssey IR dye antibodies $(1: 14,000)$ and visualized with an Odyssey IR imaging system (LI-COR Biosciences).

\section{RNA-seq library preparation}

Cells were lysed in Trizol and total RNA was extracted using the Direct-zol kit (Zymo Research, CA USA). On column DNA digestion was also performed with DNAse treatment. Poly(A) RNA was selected using the NEBNext Poly(A) mRNA Magnetic Isolation module (New England Biolabs) and libraries were prepared using the NEBNext Ultra Directional RNA Library Prep Kit (New England Biolabs) and sequenced using a NextSeq 500 (Illumina). Samples were sequenced at a minimum depth of 15 million reads per sample, paired end with a read length of $2 \times 41 \mathrm{bp}$.

\section{RNA sequencing data analysis}

A computational pipeline was written calling scripts from the CGAT toolkit to analyse the RNA sequencing data (https://github.com/cgat-developers/cgat-flow) ${ }^{4,5}$. Briefly, FASTQ files were generated and assessed for quality using FASTQC, aligned to GRCh38 (hg38) and then aligned to the transcriptome using hisat2 v2.1.0 ${ }^{6}$. To count mapped reads to individual genes, featurecounts v1.4.6, part of the subreads package ${ }^{7}$, was used. Differential gene expression analysis was performed using DESeq2 using treatment and time as factors in the model. Genes were considered to be differentially expressed based on log 2 fold change and $p$-value $<0.05$. $R$ scripts used to analyze the transcriptomic data are available through GitHub (https://github.com/Acribbs/deseq2 report). Motif enrichment was performed using homer as described before ${ }^{8,9}$. 


\section{Preparation and infection by pseudotyped VSV}

Vesicular Stomatitis Virus (VSV) pseudotyped with spike proteins of SARS-CoV-2 were generated according to a published protocol ${ }^{10}$. Briefly, HEK293T, transfected to express full length SARSCoV-2 spike proteins, were inoculated with VSV-G pseudotyped $\Delta$ G-luciferase or GFP VSV (Kerafast, MA). After $2 \mathrm{hr}$ at $37^{\circ} \mathrm{C}$, the inoculum was removed and cells were refed with DMEM supplemented with $10 \%$ FBS, $50 \mathrm{U} / \mathrm{mL}$ penicillin, $50 \mu \mathrm{g} / \mathrm{mL}$ streptomycin, and VSV-G antibody (I1, mouse hybridoma supernatant from CRL-2700; ATCC). Pseudotyped particles were collected $20 \mathrm{hr}$ post-inoculation, centrifuged at $1,320 \times \mathrm{g}$ to remove cell debris and stored at $-80^{\circ} \mathrm{C}$ until use. Cells were seeded at 10,000 cells per well in a 96-well plate. The cells were then treated with Halufuginone for $16 \mathrm{hrs}$. As a control for HS dependent infection some cells were treated with HSases for 30 min at $37^{\circ} \mathrm{C}$ in serum-free DMEM. Culture supernatant containing pseudovirus $(20-100 \mu \mathrm{L})$ was adjusted to a total volume of $100 \mu \mathrm{L}$ with PBS, HSase mix or the indicated inhibitors and the solution was added to the cells. After $4 \mathrm{hr}$ at $37^{\circ} \mathrm{C}$ the media was changed to complete DMEM. The cells were then incubated for $16 \mathrm{hr}$ to allow expression of the luciferase gene. Cells were analyzed for infection by Bright-Glo ${ }^{\mathrm{TM}}$ (Promega) using the manufacturers protocol. Briefly, $100 \mu \mathrm{L}$ of luciferin lysis solution was added to the cells and incubated for $5 \mathrm{~min}$ at room temperature. The solution was transferred to a black 96-well plate and luminescence was detected using an EnSpire multimodal plate reader (Perkin Elmer). Data analysis and statistical analysis was performed in Prism 8.

\section{SARS-CoV-2 infection}

SARS-CoV-2 isolate USA-WA1/2020 (BEI Resources) was propagated and infectious units quantified by plaque assay using Vero E6 (ATCC) cells. Approximately 10e4 Vero E6 cells per well were seeded in a 96 well plate and incubated overnight. The following day, cells were washed with PBS and 100uL of SARS-CoV-2 (MOI 0.5) diluted in serum free DMEM was added per well and incubated $1 \mathrm{~h}$ at $37^{\circ} \mathrm{C}$ with rocking every $10-15 \mathrm{~min}$. After $1 \mathrm{~h}$, virus was removed, cells 
washed with PBS and compounds or controls were added at the indicated concentrations. In experiments using inhibitors of the ISR or its related kinases, these compounds were added after viral infection and incubated for $1 \mathrm{~h}$ prior to the addition of HF. For viral RNA quantification, cells were washed twice with PBS and lysed in 200ul TRIzol (ThermoFisher). For immunofluorescence, cells were washed twice with PBS and incubated in $4 \%$ formaldehyde for 30 minutes at room temperature. For plaque assays, supernatant was removed and stored at -80 until plaque assays were performed.

\section{Flavivirus and alphavirus infection}

Sindbis virus (SINV) with mCherry-tagged NSP3 was propagated on BHK-15 cells ${ }^{11}$. Uganda strain of Zika virus (ZIKV) MR 766 encoding the fluorescent protein Venus from the genome (a kind gift from Matthew Evans, Schwarz 2016) was propagated on Huh 7.5 cells $^{12}$. Huh 7.5 and BHK-15 monolayers were pretreated with various halofuginone concentrations for 16 hours and infected with SINV or ZIKV at MOI of 0.1 for two hours in the presence of the compound. Subsequently, media over cells were replaced with fresh media containing halofuginone and incubated for 12 hours for SINV and 24 hours for ZIKV. The cell culture supernatants were collected at 12 hours post infection for SINV and 24 hours post infection for ZIKV and the virus titers were determined by fluorescent focus assay (FFT).

\section{Methods for human bronchial epithelial cell ALI generation and infection}

\section{Air Liquid Interface}

Human Bronchial Epithelial Cells (HBECs, Lonza) were cultured in T75 flasks in PneumaCult-Ex Plus Medium according to manufacturer instructions (StemCell Technologies). To generate airliquid interface cultures, HBECs were plated on collagen I-coated 24 well transwell inserts with a 0.4-micron pore size (Costar, Corning) at $5 \times 10^{4}$ cells $/ \mathrm{ml}$. Cells were maintained for 3-4 days in PneumaCult-Ex Plus Medium until confluence, then changed to PneumaCult-ALI Medium 
(StemCell Technologies) containing ROCK inhibitor (Y-27632, Tocris) for 4 days. Fresh medium, $100 \mu \mathrm{l}$ in the apical chamber and $500 \mu \mathrm{l}$ in the basal chamber, was added daily. At day 7 , the medium in the apical chambers was removed, and the basal chambers were changed every 2-3 days with apical washes with PBS every week for 28 days.

Human bronchial epithelial cell ALI Infection.

The apical side of the HBEC ALI culture was gently washed three times with $200 \mu$ l of phosphate buffered saline without divalent cations (PBS-/-). An MOI of 0.5 of SARS-CoV-2 live virus in 100 $\mu l$ total volume of PBS was added to the apical chamber with either DMSO, Heparinase or or various halofuginone concentrations. Cells were incubated at $37 \circ \mathrm{C}$ and $5 \% \mathrm{CO} 2$ for 4 hours. Unbound virus was removed, the apical surface was washed and the compounds were re-added to the apical chamber. Cells were incubated for another 20 hours at $37 \circ \mathrm{C}$ and $5 \% \mathrm{CO} 2$. After inoculation, cells were washed once with PBS-/- and $100 \mu$ l TrypLE (ThermoFisher) was added to the apical chamber then incubated for $10 \mathrm{~min}$ in the incubator. Cells were gently pipetted up and down and transferred into a sterile $15 \mathrm{ml}$ conical tube containing neutralizing medium of DMEM $+3 \%$ FBS. TrypLE was added again for 3 rounds of 10 minutes for a total of 30 min to clear transwell membrane. Cells were spun down and resuspended in PBS with Zombie UV viability dye for $15 \mathrm{~min}$ in room temp. Cells were washed once with FACS buffer then fixed in $4 \%$ PFA for 30 min at room temp. PFA was washed off and cells were resuspended in PBS.

\section{Virus plaque assays}

Confluent monolayers of Vero E6 or Hep3B cells were infected with SARS-CoV-2 at an MOI of 0.1. After one hour of incubation at $37^{\circ} \mathrm{C}$, the virus was removed, and the medium was replaced. After $48 \mathrm{hr}$, cell culture supernatants were collected and stored at $-80^{\circ} \mathrm{C}$. Virus titers were determined by plaque assays on Vero E6 monolayers. In short, serial dilutions of virus stocks in Dulbecco's Modified Essential Media (DMEM) (Corning, \#10-014-CV) were added to Vero E6 monolayers on 12 -well plates and incubated $1 \mathrm{hr}$ at $37^{\circ} \mathrm{C}$ with rocking every $10-15 \mathrm{~min}$. The cells 
were subsequently overlaid with MEM containing $0.6 \%$ agarose (ThermoFisher Scientific, \#16500-100), 4\% FBS, non-essential amino acids, L glutamine, and sodium bicarbonate and the plates were incubated at $37{ }^{\circ} \mathrm{C}$ under an atmosphere of $5 \% \mathrm{CO}_{2} / 95 \%$ air for $48 \mathrm{hr}$. The plates were fixed with a mixture of $10 \%$ formaldehyde and $2 \%$ methanol (v/v in PBS) for $24 \mathrm{hr}$. Agarose overlays were removed, and the monolayer was washed once with PBS and stained with $0.025 \%$ Crystal Violet prepared in $2 \%$ ethanol. After $15 \mathrm{~min}$, Crystal Violet was removed, and plaques were counted to determine the virus titers. Plaque assays were performed and counted by a blinded experimenter. All work with SARS-CoV-2 was conducted in Biosafety Level-3 conditions either at the University of California San Diego or at the Eva J Pell Laboratory, The Pennsylvania State University, following the guidelines approved by the Institutional Biosafety Committees.

\section{RNA extraction, cDNA synthesis and qPCR:}

RNA was purified from TRIzol lysates using Direct-zol RNA Microprep kits (Zymo Research) according to manufacturer recommendations that included DNase treatment. RNA was converted to cDNA using the iScript cDNA synthesis kit (BioRad) and qPCR was performed using iTaq universal SYBR green supermix (BioRad) and an ABI 7300 real-time pcr system. cDNA was amplified using the following primers RPLPO F - GTGTTCGACAATGGCAGCAT; RPLPO R GACACCCTCCAGGAAGCGA; $\quad$ SARS-CoV-2 $\quad$ Spike $\quad$ F _ CCTACTAAATTAAATGATCTCTGCTTTACT; $\quad$ SARS-CoV-2 $\quad$ Spike $\quad \mathrm{R}$ CAAGCTATAACGCAGCCTGTA. Relative expression of SARS-CoV-2 Spike RNA was calculated by delta-delta-Ct by first normalizing to the housekeeping gene RPLPO and then comparing to SARS-CoV-2 infected Vero E6 cells that were untreated (reference control). Curves were fit and inhibitory concentration (IC) IC50 and IC90 values calculated using Prism 8.

\section{Immunofluorescence imaging and analysis}


Formaldehyde fixed cells were washed with PBS and permeabilized for immunofluorescence using BD Cytofix/Cytoperm according to the manufacturers protocol for fixed cells and stained for SARS-CoV-2 with a primary anti-Nucleocapsid antibody (GeneTex GTX135357) followed by a secondary Goat anti Rabbit AF594 antibody (ThermoFisher A-11037) and nuclei stained with Sytox Green. Five or eight images per well were obtained using an Incucyte S3 (Sartorius) or Nikon Ti2-E microscope equipped with a Qi-2 camera and Lumencor Spectra III light engine respectively. The percent infected cells were calculated using built-in image analysis tools for the Incucyte S3. For images acquired with the Nikon Ti2, images were analysed using the Fiji distribution of ImageJ (PMID 22743772) and the DeepLearning plugin StarDist (Schmidt et al, 2012 - see below) as follows. Channels were separated and the Sytox Green-stained nuclei were segmented using StarDist to generate individual masks for each nucleus. The AF594 channel (Nucleocapsid) was selected by removing background with a rolling ball of 50 pixels. A median filter (sigma=10) was applied to facilitate easier thresholding of AF594 signal. The resulting image was thresholded and a mask was generated representing all positive staining. Positive nuclei were selected by firstly eroding the StarDist generated nuclei mask by 2 pixels to reduce potential overlap with non-positive stain. Binary reconstruction was then carried out between the resulting mask and the AF594 mask. Uwe Schmidt, Martin Weigert, Coleman Broaddus, and Gene Myers. Cell Detection with Star-convex Polygons. International Conference on Medical Image Computing and Computer-Assisted Intervention (MICCAI), Granada, Spain, September 2018.

\section{Proline distribution analysis}

Protein amino acid sequences were downloaded from UniProtKB ${ }^{13}$. Proline distribution was analyzed using custom code in $\mathrm{R}$ (v3.6.0) ${ }^{14}$, including commands from the packages dplyr ${ }^{15}$ tidyr ${ }^{16}$, and stringr ${ }^{17}$. All plots were generated in ggplot2 ${ }^{18}$. For individual protein plots, histograms were constructed with geom_histogram(binwidth =1) and kernel density estimations (KDEs) were constructed with geom_density(kernel = "gaussian") and added as custom annotations (ggpubr 
package $\left.{ }^{19}\right)$. The bandwidth of KDEs for individual plots was assessed separately for each protein distribution using maximum likelihood cross-validation ${ }^{20,21}$ with the h.mlcv command from the kedd package ${ }^{22}$. The proline distribution score was calculated using the following formula:

$$
\text { Proline dist. score }=\log _{10}\left(B_{P} /_{B_{T}} * P\right)
$$

where $B_{P}$ is the number of 10 -amino acid blocks in a protein that contain one or more prolines (a protein with length 100 amino acids has 10 blocks); $B_{T}$ is the total number of 10 -amino acid blocks in a protein; and $\mathrm{P}$ is the total number of prolines in a protein. $\mathrm{B}_{\mathrm{P}}$ values were obtained using custom code in $\mathrm{R}$.

\section{Data and Code availability}

Data are deposited in the National Centre for Biotechnology Information GEO database under the accession number (GSE157036). Depicted structures have been solved and deposited: halofuginone PDB ID 4K88, ProSA PDB ID 5V58, and NCP22 PDB ID 5VAD. The custom-scripted macro used for automated image analysis of live cell imaging data is available from https://doi.org/10.26180/5f508284eb365. Code and files used for proline analysis can be found on https://github.com/jkccoker/Proline analysis. 


\section{References for Methods}

1 Blight, K. J., McKeating, J. A. \& Rice, C. M. Highly permissive cell lines for subgenomic and genomic hepatitis C virus RNA replication. J Virol 76, 13001-13014, doi:10.1128/jvi.76.24.13001-13014.2002 (2002).

2 Anower, E. K. F., Singh, G., Deng, Y., Gordts, P. \& Esko, J. D. Corrigendum to: Triglyceride-rich lipoprotein binding and uptake by heparan sulfate proteoglycan receptors in a CRISPR/Cas9 library of Hep3B mutants. Glycobiology 30, 760, doi:10.1093/glycob/cwaa016 (2020).

3 Esko, J. D. in Current protocols in molecular biology (eds F. Ausubel et al.) 17.12.1117.12.19 (Greene Publishing and Wiley-Interscience, 1993).

4 Cribbs AP, L.-V. S., George C et al. CGAT-core: a python framework for building scalable, reproducible computational biology workflows. F1000Research 8:377 (2019).

5 Sims, D. et al. CGAT: computational genomics analysis toolkit. Bioinformatics 30, 12901291, doi:10.1093/bioinformatics/btt756 (2014).

6 Kim, D., Paggi, J. M., Park, C., Bennett, C. \& Salzberg, S. L. Graph-based genome alignment and genotyping with HISAT2 and HISAT-genotype. Nat Biotechno/ 37, 907-915, doi:10.1038/s41587-019-0201-4 (2019).

7 Liao, Y., Smyth, G. K. \& Shi, W. The R package Rsubread is easier, faster, cheaper and better for alignment and quantification of RNA sequencing reads. Nucleic Acids Res 47, e47, doi:10.1093/nar/gkz114 (2019).

8 Heinz, S. et al. Simple combinations of lineage-determining transcription factors prime cisregulatory elements required for macrophage and B cell identities. Mol Cell 38, 576-589, doi:10.1016/j.molcel.2010.05.004 (2010).

9 Gordts, P. et al. Reducing macrophage proteoglycan sulfation increases atherosclerosis and obesity through enhanced type I interferon signaling. Cell Metab 20, 813-826, doi:10.1016/j.cmet.2014.09.016 (2014). 
10 Whitt, M. A. Generation of VSV pseudotypes using recombinant DeltaG-VSV for studies on virus entry, identification of entry inhibitors, and immune responses to vaccines. $J$ Virol Methods 169, 365-374, doi:10.1016/j.jviromet.2010.08.006 (2010).

11 Jose, J., Taylor, A. B. \& Kuhn, R. J. Spatial and Temporal Analysis of Alphavirus Replication and Assembly in Mammalian and Mosquito Cells. mBio 8, doi:10.1128/mBio.02294-16 (2017).

12 Schwarz, M. C. et al. Rescue of the 1947 Zika Virus Prototype Strain with a Cytomegalovirus Promoter-Driven cDNA Clone. mSphere 1, doi:10.1128/mSphere.00246-16 (2016).

13 UniProt, C. UniProt: a worldwide hub of protein knowledge. Nucleic Acids Res 47, D506D515, doi:10.1093/nar/gky1049 (2019).

14 Team, R. C. R: A language and environment for statistical computing. . R Foundation for Statistical Computing, Vienna, Austria. (2019).

15 H. Wickham, R. F., L. Henry, K. Müller. dplyr: A Grammar of Data Manipulation. R package version 0.8. https://CRAN.R-project.org/package=dplyr (2020).

16 Wickham, H. tidyr: Tidy Messy Data. R package version 1.1.2. https://CRAN.Rproject.org/package=tidyr (2020).

17 Wickham, H. stringr: Simple, Consistent Wrappers for Common String Operations. R package version 1.4.0. https://CRAN.R-project.org/package=stringr (2019).

18 Wickham, H. ggplot2: Elegant Graphics for Data Analysis. Springer-Verlag New York. ISBN 978-3-319-24277-4, https://ggplot2.tidyverse.org (2016).

19 Kassambara, A. ggpubr: 'ggplot2' Based Publication Ready Plots. Package version 0.4.0. https://CRAN.R-project.org/package=ggpubr (2020).

20 J.D.F. Habbema, J. H., and K. Van den Broek. A stepwise discrimination analysis program using density estimation. Compstat 1974: Proceedings in Computational Statistics, Physica Verlag, Vienna (1974). 
21 Duin, R. P. W. On the choice of smoothing parameters of Parzen estimators of probability density functions. . IEEE Transactions on Computers C-25, 1175-1179 (1976).

22 Guidoum, A. C. kedd: Kernel estimator and bandwidth selection for density and its derivatives. R. Package version 1.0.3. $h \mathrm{htp}$ ://CRAN.R-project.org/package=kedd (2015). 

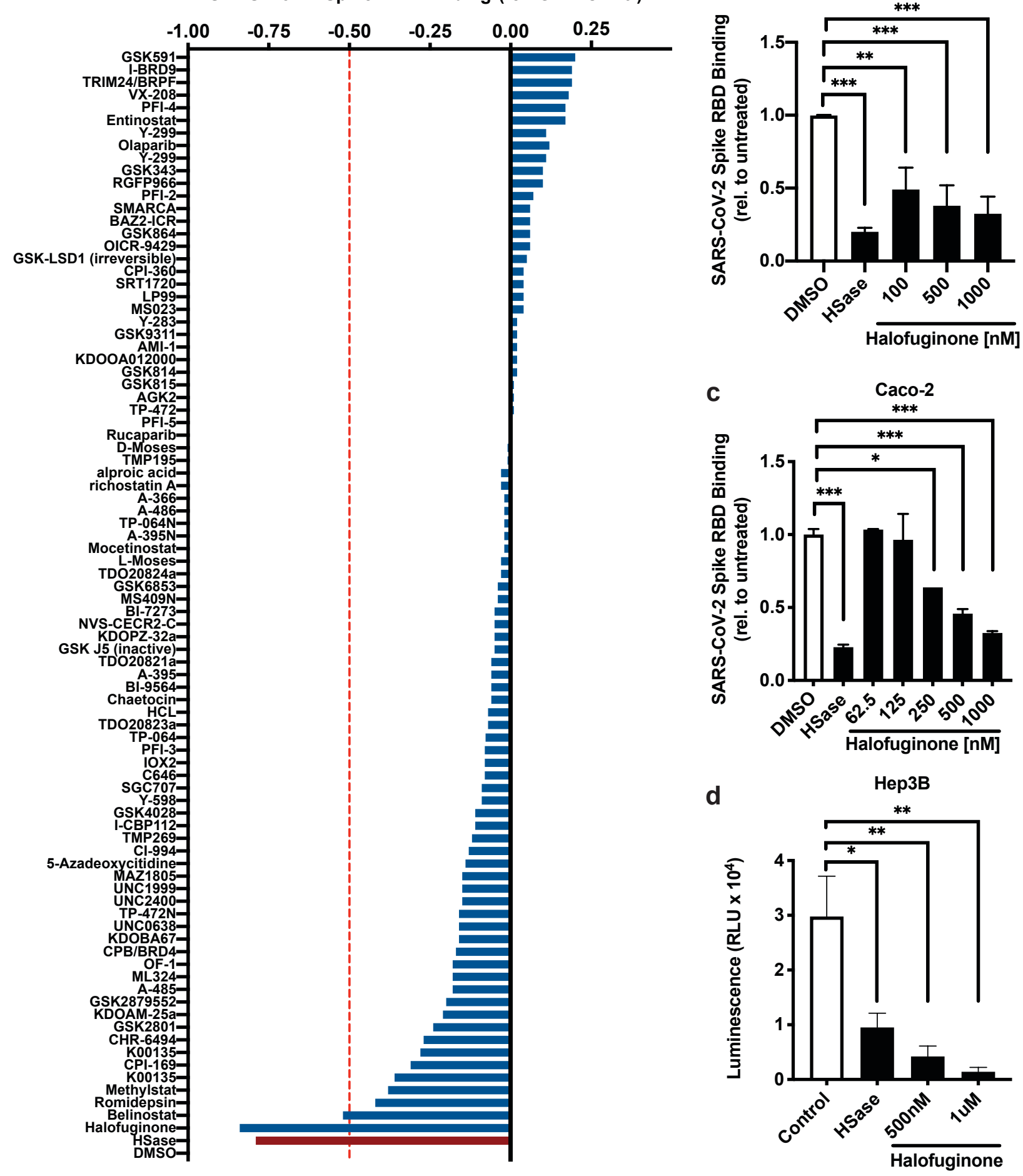
a
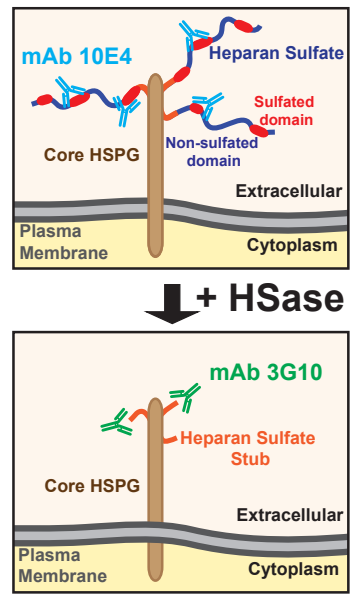

b

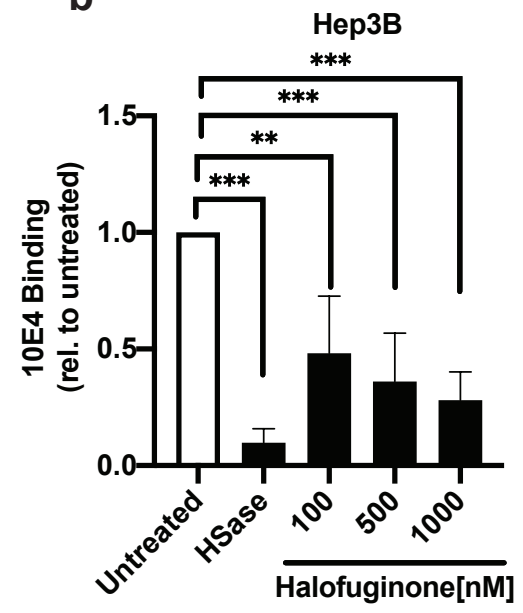

C

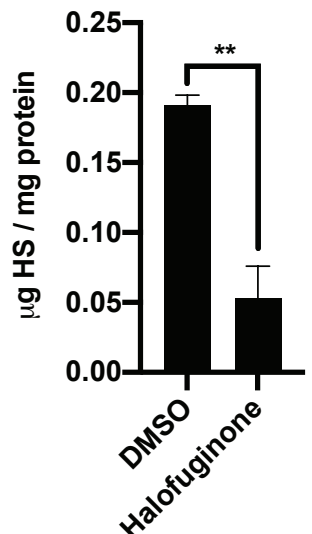

d

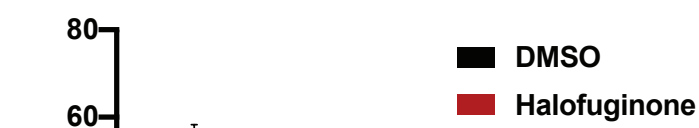

e

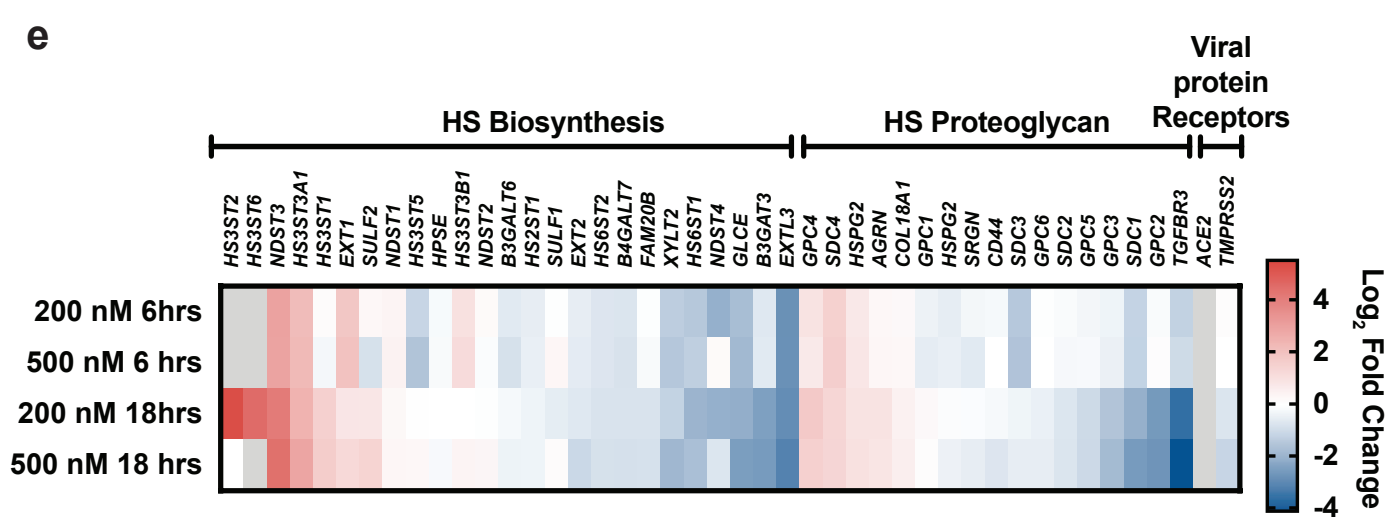

f HSase $\frac{0}{-++} \frac{500}{-++} \frac{50}{-++} \frac{5}{-++}$

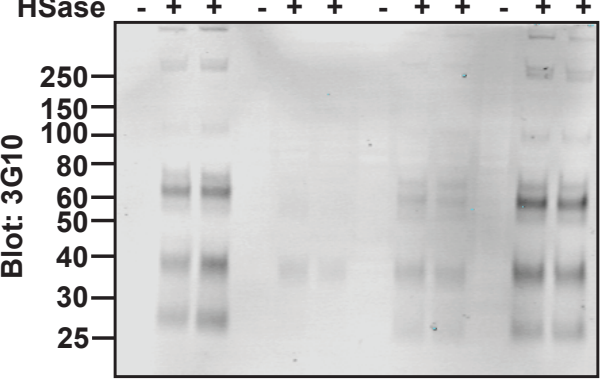

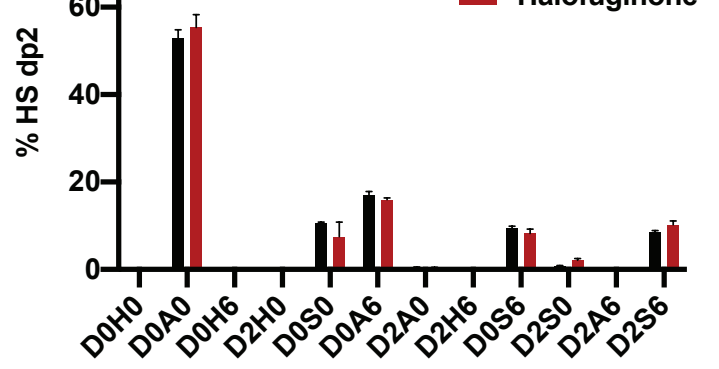

Halofuginone [nM] 
a

Halofuginone

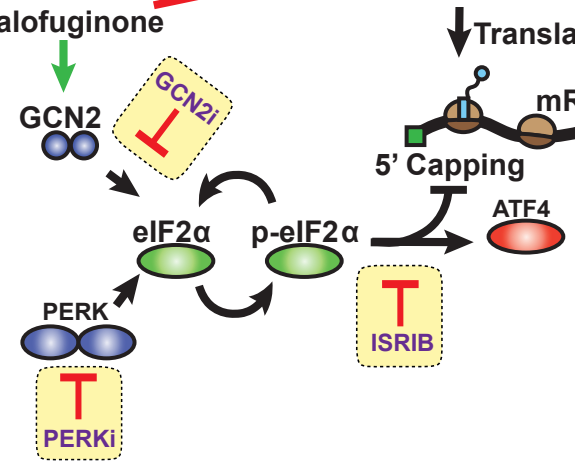

C

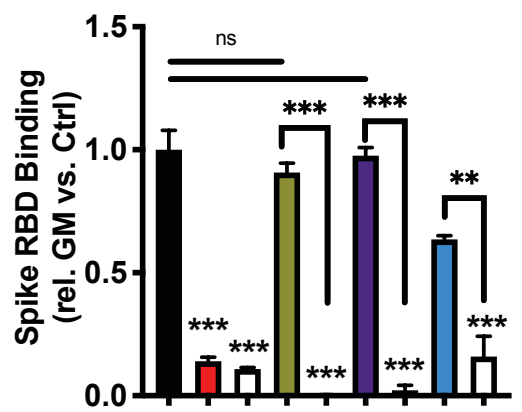

Halofuginone $--_{-}+-+++$

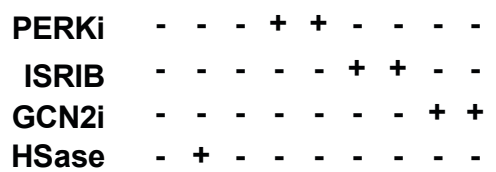

b

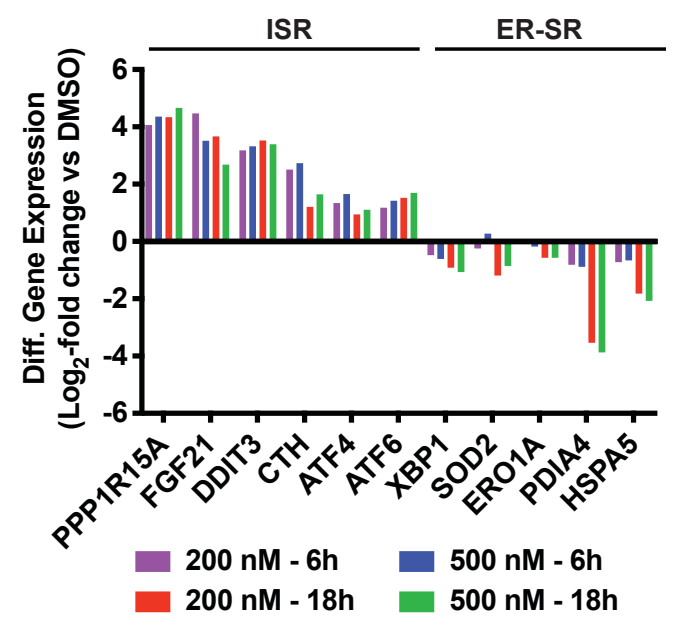

d

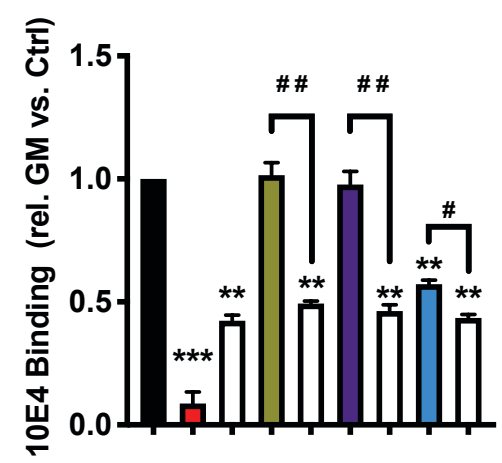

Halofuginone

PERKi

ISRIB

GCN2i

HSase 
a

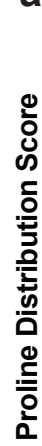

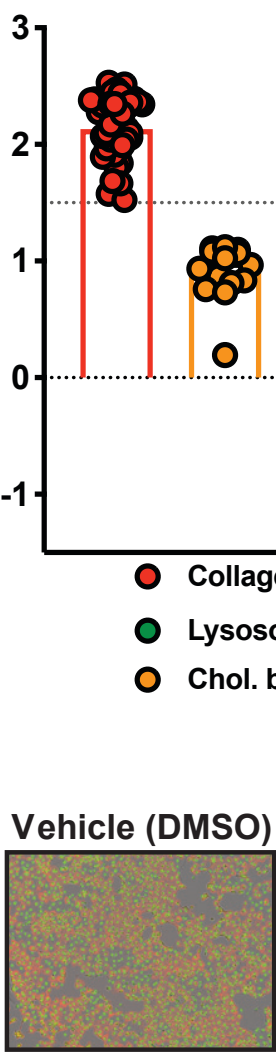

O Collagens

O SARS2 O HCoV-229E

Lysosomal

O SARS1 O HCoV-LN63

O MERS

d

b Huh7.5

C

Huh7.5
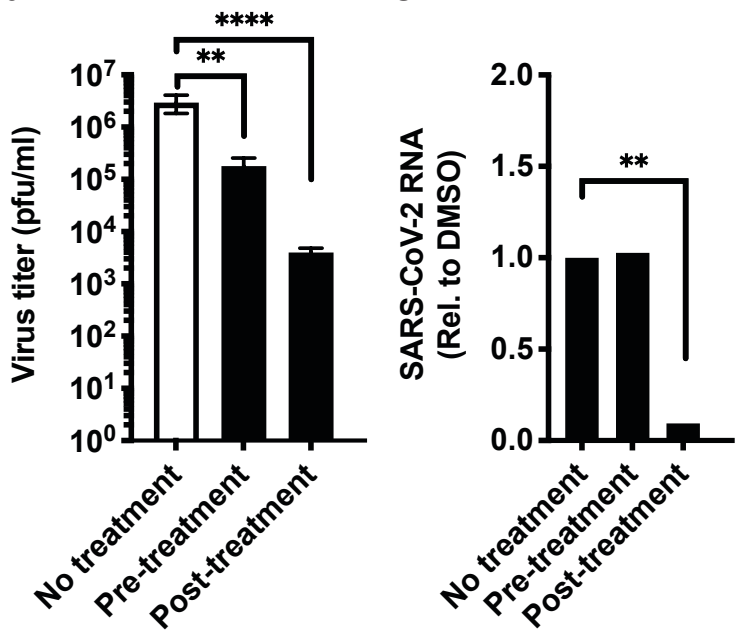

Remdesivir

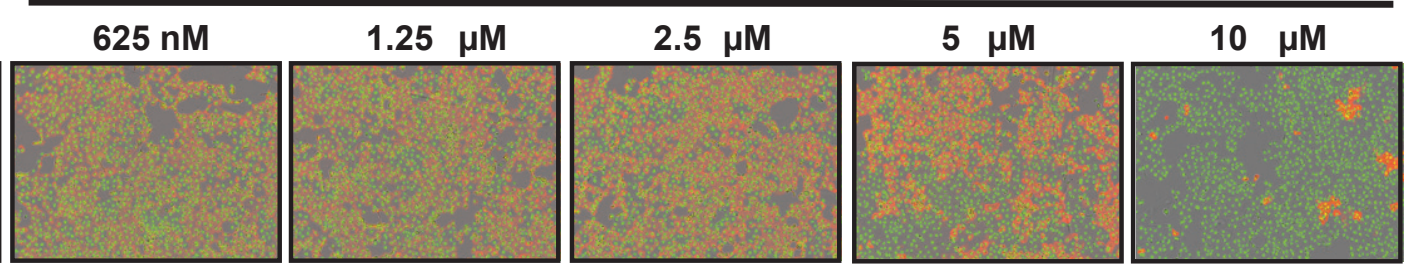

Halofuginone
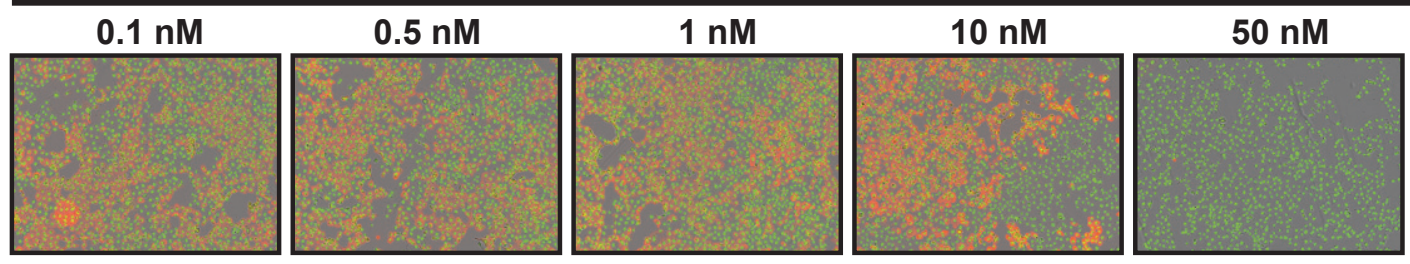

$100 \mathrm{nM}$
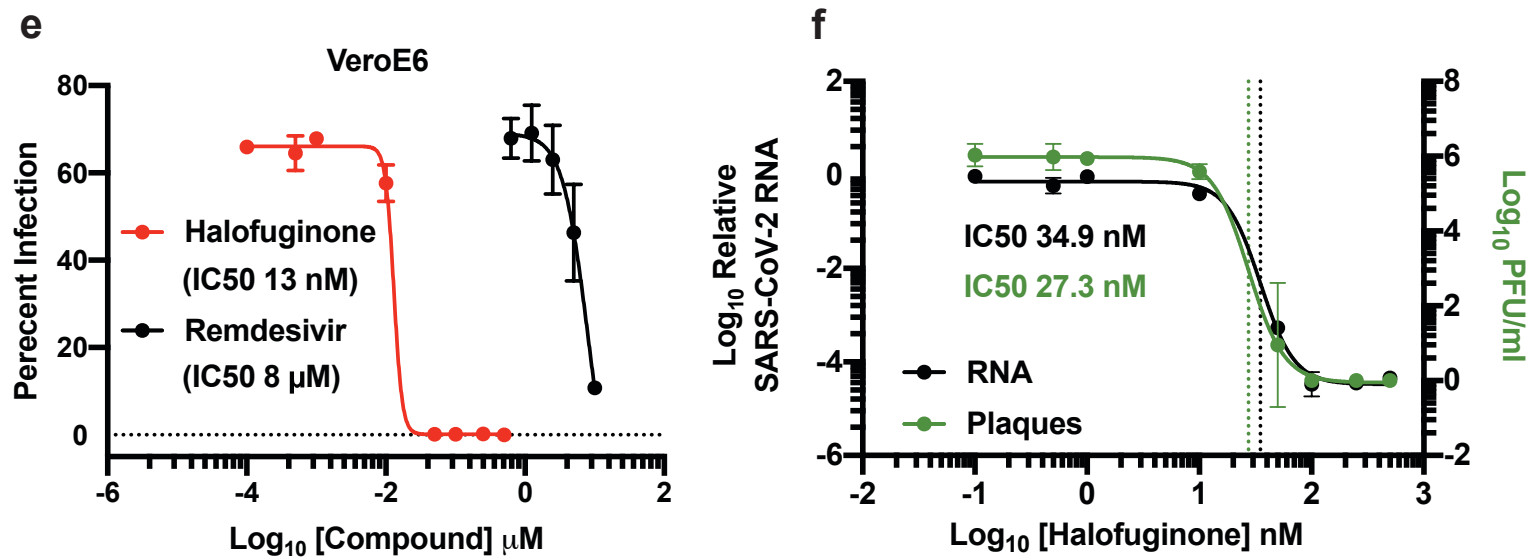
